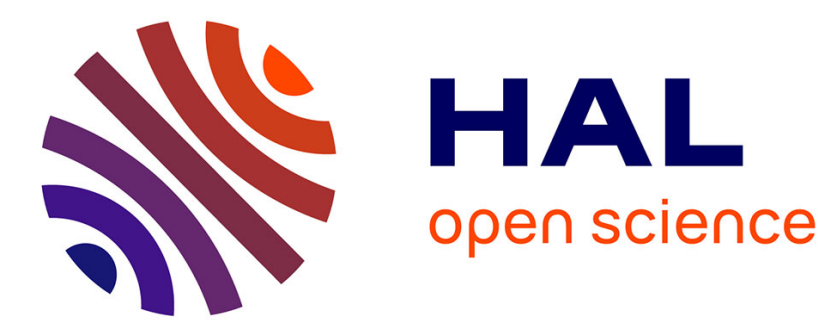

\title{
Generation and maintenance of bulk turbulence by libration-driven elliptical instability
}

\author{
Benjamin Favier, A. M. Grannan, Michael Le Bars, J. M. Aurnou
}

\section{To cite this version:}

Benjamin Favier, A. M. Grannan, Michael Le Bars, J. M. Aurnou. Generation and maintenance of bulk turbulence by libration-driven elliptical instability. Physics of Fluids, 2015, 27 (6), pp.066601. 10.1063/1.4922085 . hal-01274944

\section{HAL Id: hal-01274944 \\ https://hal.science/hal-01274944}

Submitted on 25 Oct 2018

HAL is a multi-disciplinary open access archive for the deposit and dissemination of scientific research documents, whether they are published or not. The documents may come from teaching and research institutions in France or abroad, or from public or private research centers.
L'archive ouverte pluridisciplinaire HAL, est destinée au dépôt et à la diffusion de documents scientifiques de niveau recherche, publiés ou non, émanant des établissements d'enseignement et de recherche français ou étrangers, des laboratoires publics ou privés. 


\section{AIP $\left.\right|_{\text {Fluids of }} ^{\text {Phyic }}$}

\section{Generation and maintenance of bulk turbulence by libration-driven elliptical instability}

B. Favier, A. M. Grannan, M. Le Bars, and J. M. Aurnou

Citation: Physics of Fluids 27, 066601 (2015); doi: 10.1063/1.4922085

View online: http://dx.doi.org/10.1063/1.4922085

View Table of Contents: http://scitation.aip.org/content/aip/journal/pof2/27/6?ver=pdfcov

Published by the AIP Publishing

\section{Articles you may be interested in}

Libration driven elliptical instability

Phys. Fluids 24, 061703 (2012); 10.1063/1.4729296

Sidewall boundary layer instabilities in a rapidly rotating cylinder driven by a differentially corotating lid

Phys. Fluids 22, 114109 (2010); 10.1063/1.3517292

Numerical study of eigenmode forcing effects on jet flow development and noise generation mechanisms

Phys. Fluids 21, 045106 (2009); 10.1063/1.3112686

Precession driven dynamos

Phys. Fluids 17, 034104 (2005); 10.1063/1.1852576

Numerical prediction of laminar, transitional and turbulent flows in shrouded rotor-stator systems

Phys. Fluids 9, 1696 (1997); 10.1063/1.869274

\section{Did your publisher get}

18 MILLION DOWNLOADS in 2014?

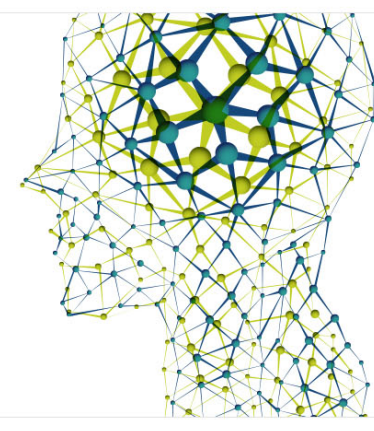




\title{
Generation and maintenance of bulk turbulence by libration-driven elliptical instability
}

\author{
B. Favier, ${ }^{1, a)}$ A. M. Grannan, ${ }^{2}$ M. Le Bars, ${ }^{1}$ and J. M. Aurnou ${ }^{1,2}$ \\ ${ }^{1}$ Aix-Marseille Université, CNRS, École Centrale Marseille, IRPHE UMR 7342, \\ 49 rue F. Joliot-Curie, 13013 Marseille, France \\ ${ }^{2}$ Department of Earth, Planetary, and Space Sciences, University of California, Los Angeles, \\ Los Angeles, California 90095-1567, USA
}

(Received 27 February 2015; accepted 23 May 2015; published online 12 June 2015)

\begin{abstract}
Longitudinal libration corresponds to the periodic oscillation of a body's rotation rate and is, along with precessional and tidal forcings, a possible source of mechanicallydriven turbulence in the fluid interior of satellites and planets. In this study, we present a combination of direct numerical simulations and laboratory experiments, modeling this geophysically relevant mechanical forcing. We investigate the fluid motions inside a longitudinally librating ellipsoidal container filled with an incompressible fluid. The elliptical instability, which is a triadic resonance between two inertial modes and the oscillating base flow with elliptical streamlines, is observed both numerically and experimentally. The large-scale inertial modes eventually lead to small-scale turbulence, provided that the Ekman number is small enough. We characterize this transition to turbulence as additional triadic resonances develop while also investigating the properties of the turbulent flow that displays both intermittent and sustained regimes. These turbulent flows may play an important role in the thermal and magnetic evolution of bodies subject to mechanical forcing, which is not considered in standard models of convectively driven magnetic field generation. (C) 2015 AIP Publishing LLC. [http://dx.doi.org/10.1063/1.4922085]
\end{abstract}

\section{INTRODUCTION}

The role of turbulence is critical in geophysical flows as it contributes to the mixing of chemical species and temperature, can enhance the viscous dissipation of energy or lead to dynamo action, for example. A conventional approach to sustain turbulent motions in the internal fluid layers of planets or satellites is to consider the continuous action of unstable entropy or compositional gradients associated with secular cooling and solidification. While this scenario has been very successful in explaining planetary magnetic fields, ${ }^{1}$ some celestial objects might be too small for thermo-solutal convection to be the only plausible source of motion (see, for example, Le Bars et $a .^{2}$ and Dwyer et al. ${ }^{3}$ for the Moon). A complementary mechanism has been proposed and relies on large-scale mechanical forcings to drive intense turbulent motions in the interiors of planets or satellites. ${ }^{4}$ Gravitational interactions between an orbiting body and its primary partner distort the shape of both bodies and give rise to periodic mechanical forcings such as precession, tides, and libration. ${ }^{5}$ While several studies have already demonstrated the dynamo capability of the flows resulting from these forcings, ${ }^{6-8}$ our understanding of the basic properties of these turbulent flows is still lacking.

This paper focuses on longitudinal libration, where the body's rotation rate undergoes periodic oscillations about its orbital rate. ${ }^{9}$ Early works focused on the libration of axisymmetric containers such as cylinders or spheres, which can lead to centrifugal instabilities ${ }^{10-12}$ and stationary zonal flows caused by non-linear interactions in the Ekman boundary layers. ${ }^{13-17}$ In the case of nonaxisymmetric containers, the coupling between the solid boundaries and the fluid is not only viscous,

a) Author to whom correspondence should be addressed. Electronic mail: favier@irphe.univ-mrs.fr 
as in axisymmetric cases, but also of a topographic nature. Although librational forcing cannot directly excite eigenmodes of the system through a direct resonance in the inviscid case, ${ }^{18,19}$ it has been shown that three-dimensional flows can be driven by the resonance of two inertial modes with an elliptically deformed base flow, ${ }^{4,20-22}$ the so-called libration-driven elliptical instability (LDEI).

More generally, the elliptical instability ${ }^{23}$ is a resonance mechanism between a pair of normal modes of the system and the underlying strain field associated with regions of two-dimensional, elliptical streamlines. Although it has been demonstrated that the instability ultimately leads to small-scale disorder both experimentally ${ }^{24}$ and numerically, ${ }^{25,26}$ our theoretical understanding of this process is mostly limited to the initial exponential phase of the instability mechanism. ${ }^{27}$ The eventual collapse of the excited inertial modes and the properties of the small-scale flow resulting from it are still not well understood. This turbulent regime in closed geometries has been barely considered mainly for technical reasons: it is difficult to obtain reliable laboratory measurements of the small-scale flow and numerical simulations are rare due to the difficulty in considering a large-scale non-axisymmetric geometry and a small-scale turbulent flow at the same time.

In order to improve our understanding of the elliptical instability and its possible applications to geophysical flows, we focus on the properties of the turbulence generated by such an instability driven by the libration of an ellipsoidal container. Our paper builds upon the recent laboratory experimental work by Grannan et al., ${ }^{22}$ where quantitative measurements were only available for the horizontal flow in the equatorial plane. We therefore complement their experimental results with high-resolution direct numerical simulations (DNS), from which a complete three-dimensional description of the flow is available. The paper is organized as follows. The model, numerical approach, and experimental setup are described in Secs. II-IV, respectively. Our results are discussed in Sec. V, where we focus on the transition to turbulence and the properties of the turbulence itself.

\section{MODEL AND EQUATIONS}

We consider the flow of an incompressible fluid with constant kinematic viscosity $v$ inside a rigid ellipsoid whose surface is defined by the Cartesian equation

$$
\frac{x^{2}}{a^{2}}+\frac{y^{2}}{b^{2}}+\frac{z^{2}}{c^{2}}=1
$$

The equatorial ellipticity of the container is defined as

$$
\beta=\frac{a^{2}-b^{2}}{a^{2}+b^{2}} .
$$

The ellipsoid is rotating around the vertical axis $\hat{z}$ with a time-dependent frequency $\Omega$ given by

$$
\Omega(t)=\Omega_{0}+\Delta \phi \omega_{l} \sin \left(\omega_{l} t\right),
$$

where $\Omega_{0}$ is the main rotation rate, $\Delta \phi$ is the libration amplitude, and $\omega_{l}$ is the libration frequency.

In this paper, we work in a frame of reference that is attached to the walls of the container, referred to as the librating frame in the following. The solid boundaries of the ellipsoid are fixed in that frame, which is advantageous from a numerical point of view. The equations of motion in the librating frame are

$$
\begin{aligned}
\frac{\partial \boldsymbol{u}}{\partial t}+\boldsymbol{u} \cdot \nabla \boldsymbol{u}+\underbrace{2[1+\epsilon \sin (f t)] \hat{\boldsymbol{z}} \times \boldsymbol{u}}_{\text {Coriolis }}=-\nabla \Pi+E \nabla^{2} \boldsymbol{u} \underbrace{-\epsilon f \cos (f t) \hat{z} \times \boldsymbol{r}}_{\text {Poincaré }}, \\
\nabla \cdot \boldsymbol{u}=0,
\end{aligned}
$$

where we use the semi-major axis $a$ as a length scale and $\Omega_{0}^{-1}$ as a time scale. The librating frame is a non-inertial frame so that two fictitious forces appear in Eq. (4): the Coriolis force which depends on the total rotation vector $\Omega(t) \hat{z}$ and the Poincare force which depends on its time derivative. $\Pi$ is the modified pressure taking into account the time-dependent centrifugal acceleration. The Ekman number is $E=v /\left(\Omega_{0} a^{2}\right), f=\omega_{l} / \Omega_{0}$ is the dimensionless libration frequency, and $\epsilon=\Delta \phi f$ is the libration forcing parameter. We only consider the case of no-slip boundary conditions. 
TABLE I. Summary of the experimental (case Expt., corresponding to case $V$ in Grannan et al. ${ }^{22}$ ) and numerical parameters considered in this study. $E$ is the Ekman number, $\mathcal{E}$ is the number of elements, $N$ is the order of the Legendre polynomials, $f$ is the normalized libration frequency, $\epsilon$ is the libration amplitude, $U_{\mathrm{rms}}$ is the root mean square velocity defined by Eq. (11), $l_{0}$ is the integral length scale defined by Eq. (14), $R e_{L}$ and $R e_{l}$ are the large-scale and small-scale Reynolds numbers, respectively, defined by Eqs. (13) and (15), and Ro is the small-scale Rossby number defined by Eq. (16). Case $A 1$ is stable whereas all other cases are unstable.

\begin{tabular}{|c|c|c|c|c|c|c|c|c|c|c|c|}
\hline Case & $E$ & $\mathcal{E}$ & $N$ & $f$ & $\epsilon$ & $\beta$ & $U_{\mathrm{rms}}$ & $l_{0}$ & $\operatorname{Re}_{L}$ & $R e_{l}$ & Ro \\
\hline Expt. & $2 \times 10^{-5}$ & $\ldots$ & $\ldots$ & 4 & 0.8 & 0.34 & $\ldots$ & $\ldots$ & $\ldots$ & $\ldots$ & $\ldots$ \\
\hline$A 1$ & $10^{-3}$ & 1280 & 7 & 4 & 0.8 & 0.34 & $\ldots$ & $\ldots$ & $\ldots$ & $\ldots$ & $\ldots$ \\
\hline$A 2$ & $5.5 \times 10^{-4}$ & 1280 & 11 & 4 & 0.8 & 0.34 & 0.060 & 0.16 & 110 & 18 & 0.19 \\
\hline$A 3$ & $5 \times 10^{-4}$ & 1280 & 11 & 4 & 0.8 & 0.34 & 0.064 & 0.16 & 128 & 20 & 0.2 \\
\hline$A 4$ & $3.5 \times 10^{-4}$ & 3200 & 7 & 4 & 0.8 & 0.34 & 0.067 & 0.13 & 191 & 25 & 0.26 \\
\hline$A 5$ & $2 \times 10^{-4}$ & 3200 & 11 & 4 & 0.8 & 0.34 & 0.072 & 0.12 & 360 & 43 & 0.3 \\
\hline A6 & $10^{-4}$ & 3200 & 15 & 4 & 0.8 & 0.34 & 0.076 & 0.1 & 760 & 76 & 0.38 \\
\hline$A 7$ & $5 \times 10^{-5}$ & 3200 & 23 & 4 & 0.8 & 0.34 & 0.085 & 0.08 & 1700 & 136 & 0.53 \\
\hline$B 1$ & $10^{-4}$ & 3200 & 13 & 4 & 0.8 & 0.17 & 0.043 & 0.12 & 430 & 52 & 0.18 \\
\hline$B 2$ & $10^{-4}$ & 3200 & 13 & 4 & 0.8 & 0.26 & 0.060 & 0.11 & 600 & 66 & 0.27 \\
\hline$C 1$ & $10^{-4}$ & 3200 & 13 & 2.4 & 1.2 & 0.34 & 0.28 & 0.2 & 2800 & 560 & 0.7 \\
\hline
\end{tabular}

In this paper, we study this system using both experimental and numerical approaches. Our geometrical parameters are similar to the ones considered by Grannan et al. ${ }^{22}$ In particular, the ellipsoid is characterized by a fixed equatorial ellipticity of $\beta=0.34$ (see Sec. V F though), which corresponds in our dimensionless units to a semi-major axis $a=1$ and $b=0.7$ and the aspect ratio $c / b$ is equal to one. We explore Ekman numbers between $10^{-3}<E<2 \times 10^{-5}$ and consider the librating frequencies $f=4$ and $f=2.4$. In order to focus on the parametric excitation of inertial modes, we only consider cases where $f>2$ so that the direct excitation of inertial modes, for which the frequency of the forcing matches the frequency of an inertial mode, is not possible. The different simulations and experiments considered in this paper and the corresponding parameters are summarized in Table I.

\section{NUMERICAL METHOD}

We solve fully three-dimensional Eqs. (4) and (5) in their weak variational form ${ }^{28}$ with the spectral element code Nek5000 (http://nek5000.mcs.anl.gov) developed and supported by Paul Fischer and collaborators (see Fischer et $a .^{29}$ and references within). Since the spectral element method combines the geometric flexibility of finite element methods with the accuracy of spectral methods, it is particularly well adapted to our problem involving turbulent flows in complex non-axisymmetric geometries. Nek5000 has, for example, already been used in the context of tidally forced rotating flows. ${ }^{30}$ The computational domain is decomposed into $\mathcal{E}$ non-overlapping hexahedral elements, and within each element, unknown velocity and pressure are represented as the tensor-product Lagrange polynomials of the orders $N$ and $N-2$ based at the Gauss-LobattoLegendre and Gauss-Legendre points, respectively. The convergence is algebraic with increasing number of elements $\mathcal{E}$ and exponential with increasing polynomial order $N$. The number of degrees of freedom for each velocity component is scaling as $N^{3} \mathcal{E}$. For all the simulations discussed in this paper, numerical convergence was checked by fixing the number of elements $\mathcal{E}$ and increasing the degree $N$ of the polynomial decomposition. The temporal discretization in Nek5000 is based on a semi-implicit formulation in which the nonlinear and rotation terms are treated explicitly in time and all remaining linear terms are treated implicitly. Note that our solution is dealiased following the $3 / 2$ rule for an exact evaluation of quadrature of inner products for non-linear terms. The code is efficiently parallelized using MPI (Message Passing Interface) and we use up to 480 processors for the highest resolution considered in this paper. 

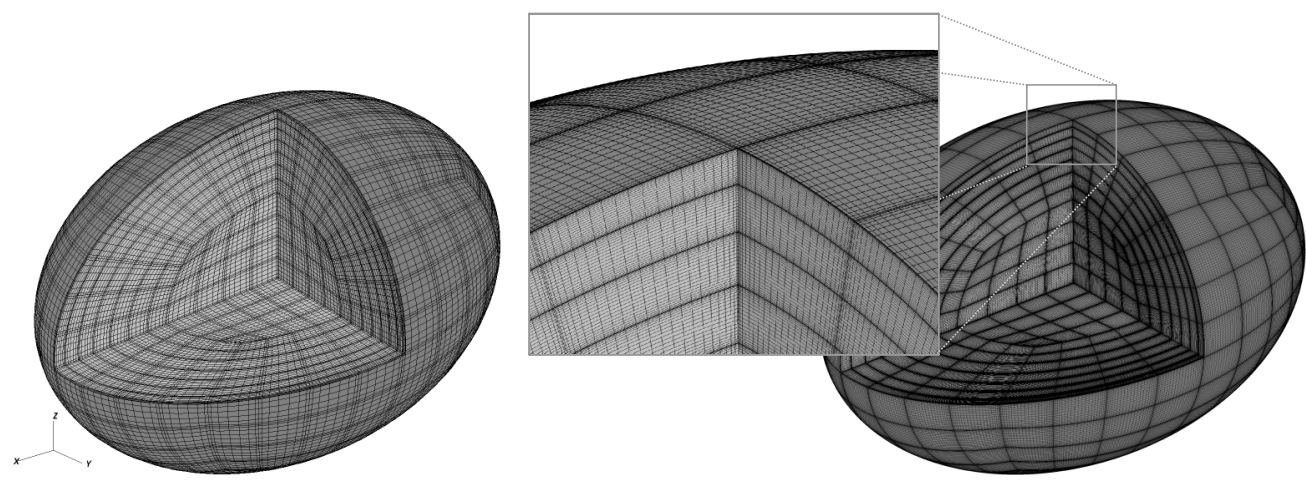

FIG. 1. Cut through the complete three-dimensional ellipsoidal mesh with 3200 elements and a polynomial order of $N=7$ (left) and $N=23$ (right). The equatorial deformation is $\beta=0.34$.

The whole ellipsoid is discretized using 1280 or 3200 elements. The mesh is a combination of a Cartesian mesh close to the origin and a spherical mesh close to the external boundary, as shown in Figure 1. The boundary geometry is initially spherical with a unit external radius centered around the origin and with a denser element distribution close to the external boundary. This, in addition to the Gauss-Lobatto-Legendre point distribution close to the element boundaries, ensures an appropriate resolution of the Ekman boundary layers with approximately ten grid points to describe them in all cases. Due to numerical limitations in terms of resolution, we can only consider flows down to $E=5 \times 10^{-5}$. The ellipsoidal grid point positions $\left(x_{e}, y_{e}, z_{e}\right)$ are obtained from the initial spherical grid points $\left(x_{s}, y_{s}, z_{s}\right)$ according to $\left(x_{e}, y_{e}, z_{e}\right)=\left(a x_{s}, b y_{s}, c z_{s}\right)$. Using this mapping approach, we can consider values of the ellipticity up to $\beta \approx 0.5$. Larger value of $\beta$ would lead to overly stretched elements with poor convergence properties, so that another type of mesh would have to be used in that case. An example of the mesh for two different polynomial orders $N$ is shown in Figure 1.

\section{EXPERIMENTAL SETUP}

The experimental setup used in the present work is adapted from the same apparatus used previously in several studies. ${ }^{10,16,22,31}$ The fluid cavity, contained within an acrylic cylinder, is ellipsoidal with a long axis $a=127 \mathrm{~mm}$ and short axes $b=c=89 \mathrm{~mm}$, leading to a fixed equatorial ellipticity of $\beta=0.34$. A first motor rotates the turntable at a constant angular velocity $\Omega_{0}=30 \mathrm{rpm}$ (corresponding to an Ekman number of $E=2 \times 10^{-5}$ for water). The second motor, situated on the turntable, is directly coupled to the acrylic container and superimposes a sinusoidal oscillation whose parameter range is $[\Delta \phi, f]=[0.05-2.5,0.5-9]$.

A particle image velocimetry (PIV) technique in the rotating reference frame is employed to obtain quantitative measurements in a horizontal plane close to the equator. The laser light sheet is horizontal and the camera is positioned above the system. PIV measurements are made only after solid body rotation has been reached and the librating forcing is turned on. The velocity field for an entire equatorial plane is resolved into a $23 \times 40$ grid with a typical spatial resolution of $8 \mathrm{~mm}$. More details about the experimental setup and results can be found in Grannan et al. ${ }^{22}$

\section{RESULTS}

In this paper, we focus mostly on the particular case where the librating frequency is $f=4$ and the libration amplitude is $\epsilon=0.8$ for both the laboratory experiment and the simulations. This corresponds to case $V$ of Grannan et al. ${ }^{22}$ The reason why we focus on the case $f=4$ is the following. The simplest determination of the mode and frequency coupling is provided by the $f=4$ case at the extreme range of the instability where we expect the participating modes to have an eigenfrequency $|\omega| \approx f / 2=2$ (although inertial modes with $|\omega|=2$ exactly do not exist in the inviscid limit, viscous modes with frequency close to but below 2 can be excited through imperfect 
resonances, as discussed in more detail in Sec. V D). Inertial modes with a dimensionless frequency $|\omega| \approx 2$ are easy to identify since their group velocity is nearly horizontal. Finally, it has been experimentally observed that the case $f=4$ leads to a sustained quasi-steady state of turbulence, ${ }^{22}$ which is easier to characterize and is the main focus of this paper. Note however that the intermittent regime is briefly discussed in Sec. V F whereas other frequencies are considered in Sec. V G.

For the numerical simulations, the Ekman number is varied from $E=10^{-3}$ down to $E=$ $5 \times 10^{-5}$, whereas the experimental setup is characterized by $E=2 \times 10^{-5}$. The simulations are initialized with a fluid at rest, but results are qualitatively the same with random velocity perturbations of small amplitude. A summary of the numerical input parameters can be found in Table I.

\section{A. General properties}

The general properties of the flows observed experimentally have already been discussed by Grannan et al.,22 so that we focus here on the new numerical results. The base flow, driven by the Poincare force and only accounting for the non-penetrative condition on the solid boundaries, is ${ }^{4,21}$

$$
\boldsymbol{U}_{b}=-\epsilon \sin (f t)[\hat{z} \times \boldsymbol{r}-\beta \nabla x y] .
$$

This flow is purely horizontal so that any departure from it, being due to viscous effects close to the boundaries or due to instabilities, will be observable in the vertical component of the velocity. We first consider the volume average of the squared vertical velocity component defined as

$$
E_{z}(t)=\frac{1}{2 V} \int_{V} u_{z}^{2} \mathrm{~d} V
$$

where $V$ is the total volume of the ellipsoid and $u_{z}$ is the vertical velocity component. Note that this quantity is not accessible in the experimental apparatus since the PIV measurements are limited to the horizontal components of the flow in the equatorial plane only. In addition to the volume average defined previously, we also consider quantities averaged over one libration period according to

$$
\bar{A}(t)=\frac{1}{T} \int_{t}^{t+T} A(\tau) \mathrm{d} \tau
$$

where $T=2 \pi / f$ is the librating period. Because the statistics discussed here evolve over hundreds of librating periods, looking at the time evolution of period-averaged quantities is much clearer since the oscillating contribution from the base flow is removed.

We show in Figure 2 the time evolution of the volume- and period-averaged squared vertical velocity $\overline{E_{z}}(t)$ for cases $A 1-A 7$ as defined in Table I. The only physical parameter that is varied between these cases is the Ekman number $E$. At very early times, the very low steady values (typically
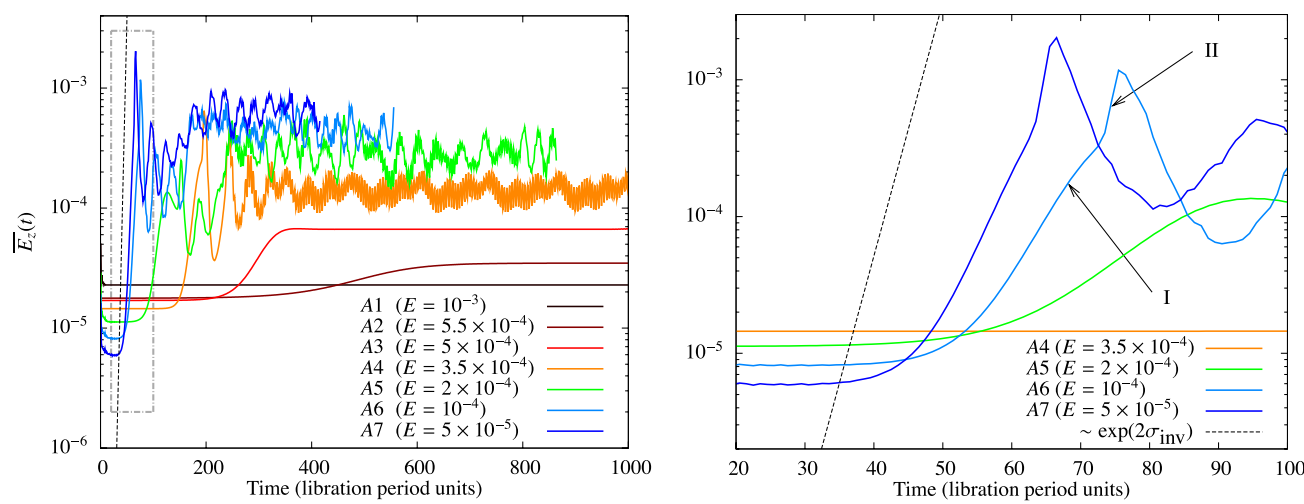

FIG. 2. Left: time evolution of the volume-averaged squared vertical velocity given by Eqs. (7) and (8) for cases $A 1-A 7$. Right: zoom on the early stage of the instability shown by the dotted contour on the left panel. The inviscid theoretical growth rate $\propto \exp \left(2 \sigma_{\text {inv }} t\right)$ as defined by Eq. (9) is plotted as a dotted line. The arrows indicate the times at which the enstrophy is visualized in Figure 3. In all cases, the results are time-averaged over each libration period according to Eq. (8). 
of order $10^{-5}$ ) of squared vertical velocity scaling as $\sqrt{E}$ are associated with the viscous corrections to the base flow given by Eq. (6) in order to match the no-slip boundary condition. Note that for the smallest Ekman number considered here (case $A 7, E=5 \times 10^{-5}$ ), we do not reach a steady base state before the instability develops. For all cases apart from case $A 1\left(E=10^{-3}\right)$, we then observe an exponential growth rate followed by a nonlinear overshoot and a steady or quasi-steady saturation of the squared vertical velocity. Case $A 1$ is stable and we do not observe any modifications of the base flow even after thousands of librating periods. It has been previously shown that this exponential growth phase is associated with the elliptical instability. ${ }^{21,22}$ A Wentzel-Kramers-Brillouin local stability analysis, valid in the regimes $\epsilon \ll 1$ and $\beta \ll 1$, leads to the following inviscid growth rate for the libration-driven elliptical instability: ${ }^{32,33}$

$$
\sigma_{\text {inv }}=\frac{16+f_{\text {res }}^{2}}{64} \epsilon \beta,
$$

where $f_{\text {res }}$ is the resonant forcing frequency at which the frequencies of the two inertial modes involved exactly follow the relation $\left|\omega_{1}-\omega_{2}\right|=f_{\text {res }}$. In the general viscous case and away from perfect resonances (i.e., $f \neq f_{\text {res }}$ ), the growth rate is reduced and given by ${ }^{20}$

$$
\sigma=\sqrt{\sigma_{\text {inv }}^{2}-\left(f_{\text {res }}-f\right)^{2}}-K \sqrt{E},
$$

where $K$ is a constant factor of order unity. The last term on the right-hand side of Eq. (10) is due to viscous dissipation in the Ekman boundary layers and is the reason why we have to consider sufficiently large values of $\beta$ and $\epsilon$ since we are limited to moderately low values of the Ekman number. As expected from these theoretical predictions, the growth rate of the instability observed in Figure 2 increases as the Ekman number decreases, and eventually tends towards inviscid growth rate (9) with $f_{\text {res }}=4$. The right panel in Figure 2 shows this exponential phase and compares it against the inviscid growth rate given by Eq. (9) (multiplied by two since we consider the squared vertical velocity). A more detailed comparison between theoretical predictions and numerical simulations can be found in Cébron et $_{\text {al. }}{ }^{21}$

After the exponential phase, the nature of the saturation depends on the Ekman number. For cases $A 2$ and $A 3$, the saturation leads to a steady-state characterized by a constant value of the volume- and period-averaged squared vertical velocity. For cases $A 4-A 7$, a quasi-steady state is obtained but significant fluctuations are observed. We also observe a strong overshoot, followed by a phase of gradual increase in the vertical energy until a quasi-steady state is eventually reached. Apart for the large $E$ cases $A 2$ and $A 3$, we also observe a low frequency modulation of the signal with a typical period of 30 librating periods. The period of these low-frequency oscillations does not depend on the Ekman number for the values considered here. Finally, note that the amplitude of saturation of the instability increases as the Ekman number decreases. This has already been discussed in Cébron et al. ${ }^{21,33}$ and our results are consistent with their conclusions, where the amplitude $A$ of the saturation scales as the square-root of the distance to the threshold $A \approx \sqrt{E_{c} / E-1}$, where $E_{c}$ is the critical Ekman number below which the elliptical instability grows.

A volume rendering of the enstrophy is shown in Figure 3 (Multimedia view) for the case $A 6$ with $E=10^{-4}$. We consider three different times: an arbitrary time during the exponential phase, just before the first overshoot and, finally, during the quasi-steady state. During the exponential phase, the flow is characterized by three components: the base flow given by Eq. (6), the zonal flow (discussed in Sec. V C), and the inertial modes. The layered structures observed in the left panel of Figure 3 correspond to the dominant $|\omega| \lesssim 2, m=1$ inertial modes resonating with the base flow. When the instability first saturates (see Figure 3 II), a sudden wave breaking event occurs, leading to intense three dimensional motions. Finally, in the quasi-steady state, a sustained inhomogeneous state of bulk turbulence is observed. The initial collapse of the inertial modes and the quasi-steady saturated state are best visualized by the two movies in Figure 3 (Multimedia view).

We now define various dimensionless numbers to describe the nature of our solutions. The typical velocity of the small-scale flow is estimated as

$$
U_{\mathrm{rms}}=\sqrt{\left\langle\left(\boldsymbol{u}-\boldsymbol{U}_{b}\right)^{2}\right\rangle_{\text {bulk }}}
$$



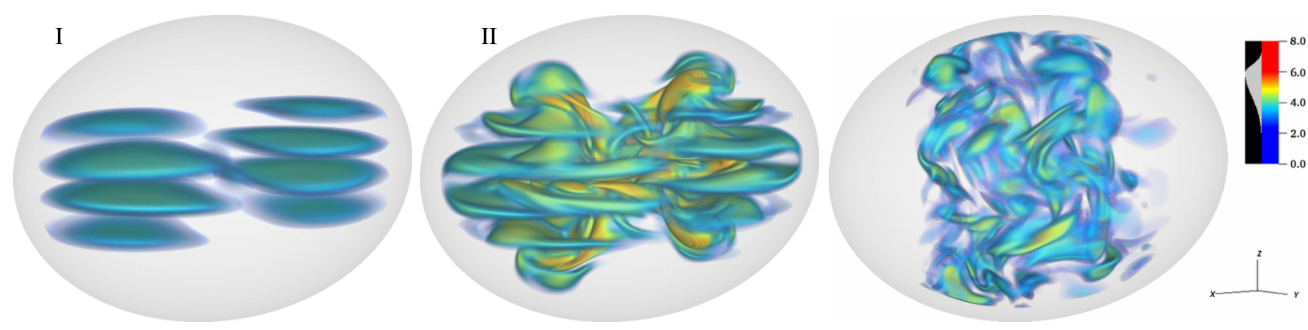

FIG. 3. Volume rendering of the enstrophy in the bulk of the ellipsoid (the boundary layers are removed from the visualization) for case $A 6$. We visualize the flow just during the exponential growth of the instability (left, $t=68$, see $\mathrm{I}$ in Figure 2), during the first collapse (middle, $t=75$, see II in Figure 2), and during the quasi-steady saturated state (right, $t=200$ ). A first movie is available as a supplement showing the collapse of the inertial modes from $t \approx 68$ to $t \approx 78$ in the librating frame. It can be compared with a similar movie (presented in the frame rotating at constant rate $\Omega_{0}$ and not in the librating frame) corresponding to case $V$ in Grannan et al. ${ }^{22} \mathrm{~A}$ second movie showing the quasi-steady regime $(186<t<196)$ is also available. (Multimedia view) [URL: http://dx.doi.org/10.1063/1.4922085.1] [URL: http://dx.doi.org/ $10.1063 / 1.4922085 .2]$

where $\boldsymbol{U}_{b}$ is the base flow defined by Eq. (6) and $\langle.\rangle_{\text {bulk }}$ denotes the volume average over the bulk of the flow. Similar to Cébron et al. ${ }^{33}$ the bulk is obtained by removing the contribution from the viscous boundary layer by assuming that their thickness is of order ${ }^{13}$

$$
\delta=\sqrt{\frac{2 E}{f}} .
$$

First, a large-scale Reynolds number, based on the semi-major axis of the ellipsoid and the rootmean-square velocity, is defined using our dimensionless units as

$$
R e_{L}=\frac{U_{\mathrm{rms}}}{E} .
$$

In addition, we also quantify the small-scale Reynolds number based on the fluctuations generated by the instability. In order to measure the typical length scales associated with the fluctuations, we defined the correlation length scale of the vertical velocity in the bulk of the domain as

$$
l_{0}=\int_{0}^{r_{0}} \frac{\left\langle u_{z}(\boldsymbol{x}) u_{z}\left(\boldsymbol{x}+r \boldsymbol{e}_{i}\right)\right\rangle_{\text {bulk }}}{\left\langle u_{z}^{2}(\boldsymbol{x})\right\rangle_{\text {bulk }}} \mathrm{d} r,
$$

where the integral of the correlation function is carried out up to the first zero-crossing only. We only consider the transverse correlations (where we average over both horizontal directions) of the vertical velocity since the horizontal components are dominated by the presence of large-scale inertial modes (see Sec. V D). The small-scale Reynolds number is then defined as

$$
\operatorname{Re}_{l}=\frac{U_{\mathrm{rms}} l_{0}}{E} .
$$

Finally, the Rossby number associated with the instability is given in our dimensionless units by

$$
R o=\frac{U_{\mathrm{rms}}}{2 l_{0}} .
$$

The values of these dimensionless numbers, time-averaged during the quasi-steady saturated phase, are gathered in Table I. In all cases, the large-scale Reynolds number is very large, but note that for all cases considered in this section, we did not observe a destabilization of the boundary layers due to centrifugal instabilities, for example. The small-scale Reynolds number is however much smaller, which explains why the unstable cases $A 2$ and $A 3$ remain laminar even in the presence of the instability. As the Ekman number is decreasing further, $R e_{l}$ is rapidly increasing up to 136 for case $A 7$ which implies that the small-scale flow is in a developed turbulent state. Finally, the Rossby number is gradually increasing as the Ekman number decreases but is always smaller than unity. This indicates that the fluctuations associated with the instability are significantly affected by rotation in all the cases considered here. 


\section{B. Energetics}

Taking the dot product of the velocity with Eq. (4) divided by two leads to the equation for the total kinetic energy

$$
\frac{\partial K}{\partial t}=\underbrace{-\frac{\epsilon f \cos (f t)}{2} \int_{V} \boldsymbol{u} \cdot(\hat{z} \times \boldsymbol{r}) \mathrm{d} V}_{\mathcal{P}}-\underbrace{\frac{E}{2} \int_{V} \omega^{2} \mathrm{~d} V}_{\mathcal{D}},
$$

where $K$ is the volume averaged kinetic energy; the first term on the right-hand side is the power injected by the Poincaré force and the last term is the viscous dissipation. The advection term and the Coriolis terms do no work and the pressure contribution vanishes since the normal component of the velocity is zero at the boundary in the librating frame. In the frame rotating at constant rate $\Omega_{0}$, there is no Poincaré force since the frame rotation vector is steady. The pressure term does not vanish however, since the external boundary is moving. Figure 4 shows an example of the time evolution of both quantities $\mathcal{P}$ and $\mathcal{D}$ for case $A 6$, normalized by the averaged value of the power injected before the instability develops and temporally averaged over each libration period. As expected, the steady base flow is maintained by an exact balance between the power injected by the Poincare force and the viscous dissipation. As the instability develops, we first observe an increase in the power injected, followed by an increase in the dissipation. The peak of viscous dissipation occurs approximately 5 libration periods after the peak in power. This lag in the dissipation remains the same as the instability saturates and does not depend on the Ekman number for the parameters considered here.

We now describe the effect of the instability on the overall viscous dissipation. The volumeaveraged viscous dissipation is first estimated before the instability develops. In this case, the dissipation is mostly concentrated in boundary layers where the base flow is forced to match the no-slip boundary condition on the ellipsoid surface. Again, we use the period average defined by Eq. (8) to obtain the average dissipation over each librating period. Figure 4(b) shows the time evolution of the viscous dissipation normalized by its value during the initial state where the base flow is dominating the dynamics. The instability is characterized by a significant increase in the volume-averaged viscous dissipation. This additional dissipation takes place in the bulk of the fluid domain and is not associated with boundary layers. Again, the quasi-steady value of the viscous dissipation measured during the saturated phase increases when the Ekman number is decreasing, as discussed in Cébron et $a l .{ }^{33}$

\section{Enhanced zonal flows}

Even without the presence of the elliptical instability, librating flows are known to drive persistent zonal flows. ${ }^{11,16,17,34}$ The amplitude of this zonal flow does not depend on the Ekman number
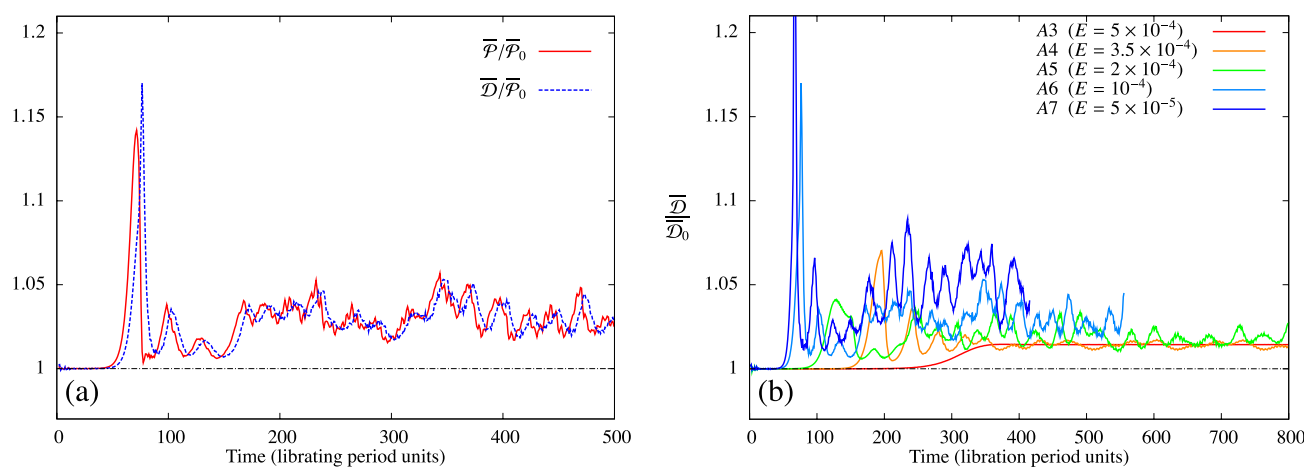

FIG. 4. (a) Time evolution of the power injected by the Poincaré force and the viscous dissipation for case $A 6$. Values are normalized by the averaged power input $\overline{\mathcal{P}}_{0}$ before the instability develops. (b) Viscous dissipation rate normalized by its value associated with the base flow only. 
and scales as $\epsilon^{2}$. Here, we discuss the effect of the libration-driven elliptical instability on such zonal flows for the case $f=4$.

We assume that the zonal flow is dominated by its azimuthal component in cylindrical coordinates and that it is mostly varying in the cylindrically radial direction. We therefore define the zonal flow as

$$
\left\langle U_{\phi}\right\rangle(\rho)=\frac{1}{N_{\rho}} \sum_{z} \sum_{\phi} \sum_{\rho-\frac{d \rho}{2}<\rho<\rho+\frac{d \rho}{2}} U_{\phi}(\rho, \phi, z),
$$

where $N_{\rho}$ is the total number of grid points lying inside the cylindrical shell and $d \rho$ is the width of the cylindrical shell. Here, we average the azimuthal flow over 30 different cylindrical shells from $\rho=0$ to $\rho=1$ and over all vertical positions $z$. In addition, these zonal flows are averaged over an arbitrary number of libration periods.

Figure 5(a) shows the radial profile of the zonal flow for different Ekman numbers. The time average is performed over two different regimes, before and after the instability kicks in. The zonal flow associated with the initial base state is shown in dotted lines, where we average the zonal profile before the exponential phase (typically for $t<200$, see Figure 2). For cases $A 3-A 5$ $\left(5 \times 10^{-4}<E<2 \times 10^{-4}\right)$, we recover the zonal flow with an amplitude independent of the Ekman number. For the cases with $E \leq 10^{-4}$ (including experimental results), the initial transient phase before the base flow is established is very long and the duration of the stable regime is too short to be able to obtain meaningful averages. As observed in previous theoretical studies, the zonal flow is prograde close to the equatorial boundary and retrograde in the bulk. The dotted-dashed line corresponds to the theoretical prediction of Sauret and LeDizès ${ }^{17}$ in the case of the sphere for $f=4$ and is shown for reference. The departure between their result and our numerical simulations is attributed to geometrical effects as we consider a non-axisymmetric container here. In particular, two persistent recirculation cells are observed in the equatorial plane (see Figure 5(b)).

When the elliptical instability saturates, the zonal flow observed in numerical simulations increases in amplitude and is now retrograde in all the bulk of the fluid (see solid lines in Figure 5(a)). Very close to the threshold (i.e., for $E>2 \times 10^{-4}$, cases $A 3$ and $A 4$ ), the amplitude of this enhanced retrograde zonal flow does not scale with the Ekman number, but for smaller Ekman numbers, the amplitude of the zonal flow increases as the Ekman number decreases. This is observed both numerically for $E=10^{-4}$ and $E=5 \times 10^{-5}$ and experimentally at $E=2 \times 10^{-5}$. Note in addition that the
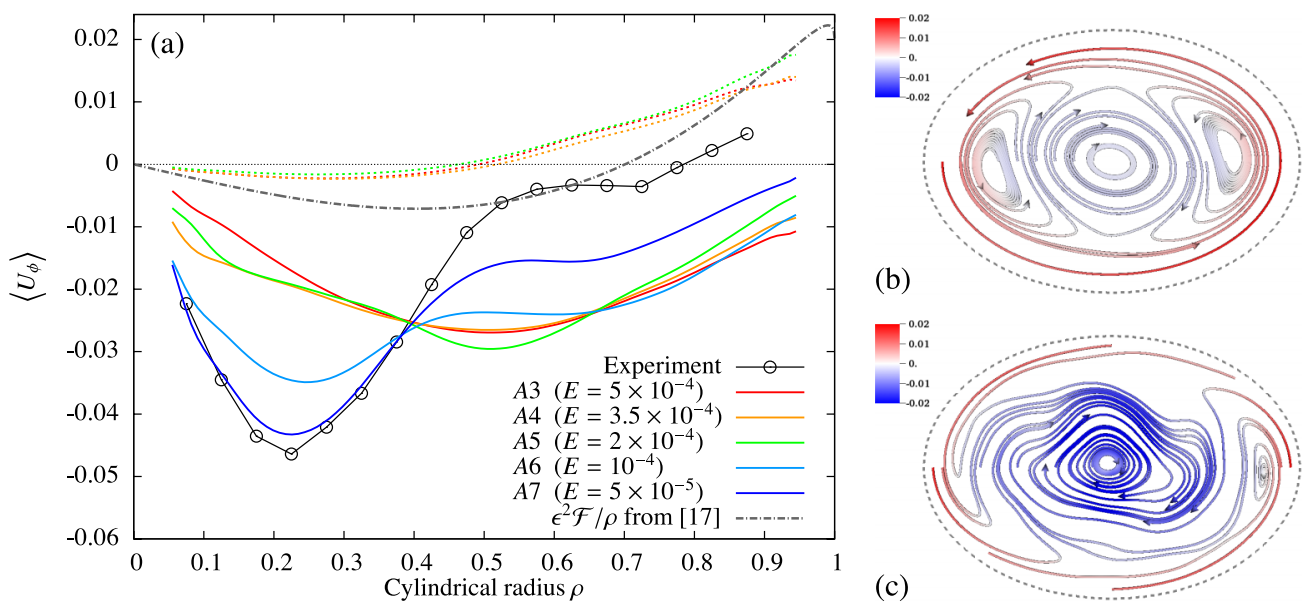

FIG. 5. (a) Comparison between the zonal flow obtained experimentally and numerically using Eq. (18) for various Ekman numbers. The dotted lines correspond to the initial steady state before the instability grows whereas the solid lines correspond to the quasi-steady state after the instability has saturated. Experimental results at $E=2 \times 10^{-5}$ are shown as empty symbols. The dashed-dotted line corresponds to theoretical results by Sauret and Le Dizès ${ }^{17}$ for the same parameters but in spherical geometry and without instabilities. Comparison of the equatorial zonal flows between the initial (b) and saturated (c) phases for case $A 6\left(E=10^{-4}\right)$. The streamlines are colored with the value of azimuthal velocity. 
trend observed in the numerical results at $E=5 \times 10^{-5}$ is consistent with the experimental results at $E=2 \times 10^{-5}$, shown as empty symbols in Figure 5(a). As the Ekman number decreases, a strong anticyclonic vortex flow develops close to axis of rotation (see Figure 5(c)), whereas its amplitude decreases close to the boundaries and eventually becomes prograde again in the experiment. Note that we observed similar behaviors for other librating frequencies such as $f=3$, so that it appears to be a generic result. Since the zonal flow associated with the base flow scales as $\epsilon^{2}$ but does not depend on the Ekman number, the relevance of such libration-driven zonal flows in geophysical systems has been questioned. ${ }^{11,17}$ Here, we show that for the particular case $f=4$, the saturation of the elliptical instability leads to an enhanced zonal flow with an amplitude increasing as the Ekman number decreases. This new nonlinearly driven zonal flow might be more relevant to the geophysical regime characterized by very low Ekman numbers. We do not have enough numerical or experimental data to provide a scaling for this mechanism at this stage, but all of our results point towards a strong retrograde axial vortex driven by nonlinearities in the turbulent bulk. Note that Mason and Kerswell ${ }^{27}$ also found a strong retrograde zonal flow driven by the saturation of the elliptical instability.

\section{Mode couplings and transition to turbulence}

In this section, we explore the transition between a laminar base flow driven by the Poincaré force and a bulk-filling turbulent flow initially driven by the elliptical instability as the Ekman number is decreased. In this study, we only considered an Ekman number of $E=2 \times 10^{-5}$ in the laboratory experiment so that the transition to turbulence occurring at larger Ekman numbers is mainly discussed from a numerical point of view. A comparison between numerical and experimental data is however presented below at the end of this section.

Before discussing the transition to turbulence in our system, let us briefly describe the spatial structures and frequencies of the inertial modes that can resonate with the harmonic forcing at $f=4$. The case of the tri-axial ellipsoid has been recently considered by Vantieghem, ${ }^{36}$ but the libration frequency $f=4$ primarily excites high wave number inertial modes and this paper mostly focuses on inertial modes with linear or quadratic spatial dependence. Following Grannan et al., ${ }^{22}$ we consider for simplicity the inviscid inertial modes in cylindrical coordinates and for a spheroidal container defined by $r^{2}+z^{2} / c^{2}=1$ as derived by Kerswell. ${ }^{35}$ The inertial modes are denoted $\Lambda_{n, m, \kappa}$, where $n$ is the order of the associated Legendre polynomials that combine the radial and axial wave numbers, $m$ is the azimuthal wave number, and $\kappa$ represents the $\kappa$ th eigenfrequency. For each couple $(n, m)$, there are $n-|m|$ eigenfrequencies (or $n-1$ if $m=0$ ). For an elliptical deformation with azimuthal wave number $m=2$, the resonance condition imposes $\left|m_{2}-m_{1}\right|=2$ where $m_{2}$ and $m_{1}$ are the azimuthal wave numbers of the inertial modes. We focus here on the case $|m|=1$ shown to be relevant by Grannan et $a l .^{22}$ Since the frequency of the forcing is $f=4$, we expect inertial modes with eigenfrequencies $|\omega| \lesssim 2$. As already mentioned, the frequency of the inertial modes cannot be exactly equal to 2 . However, an imperfect resonance is possible provided that the frequency mismatch in order to satisfy the resonance condition $\left|\omega_{1}-\omega_{2}\right|=f$ is less than the elliptical deformation $\beta .{ }^{37}$ Since we focus here on a large elliptic deformation $\beta=0.34$, resonance bands are very broad and many imperfect resonances can occur. ${ }^{38}$ Note finally that resonances were observed experimentally $^{22}$ up to $f=4+\epsilon \beta+O\left(\epsilon^{2} \beta^{2}\right)$, which is consistent with theoretical predictions in the large $\beta$ and large $\epsilon$ regimes. ${ }^{32}$ Figure 6 shows the amplitude of modes with moderately large $n$, $m=1$, and frequency close to $\omega=2$. As $n$ increases, the largest eigenfrequency $\kappa=n-|m|$ tends towards $\omega=2 \cdot{ }^{37}$ In the presence of viscosity, inertial modes with large $n$ will however be damped. Since these modes have a frequency close to $\omega=2$, their group velocity is quasi-horizontal and they are characterized by a pancake-like structure. We recall that such modes correspond to a spheroidal geometry, but we nevertheless expect the results to be similar in the ellipsoidal case. Grannan et al..$^{22}$ have, for example, reported a mode with spatial structure very similar to the spheroidal mode $\Lambda_{8,1,7}$ in their laboratory apparatus for the case $f=4$.

We now come back to the numerical results discussed in Secs. V A-V C. We place 100 numerical probes homogeneously distributed inside the bulk of the ellipsoid (i.e., outside of the viscous boundary layers). The three components of the velocity and the pressure are saved at these locations 

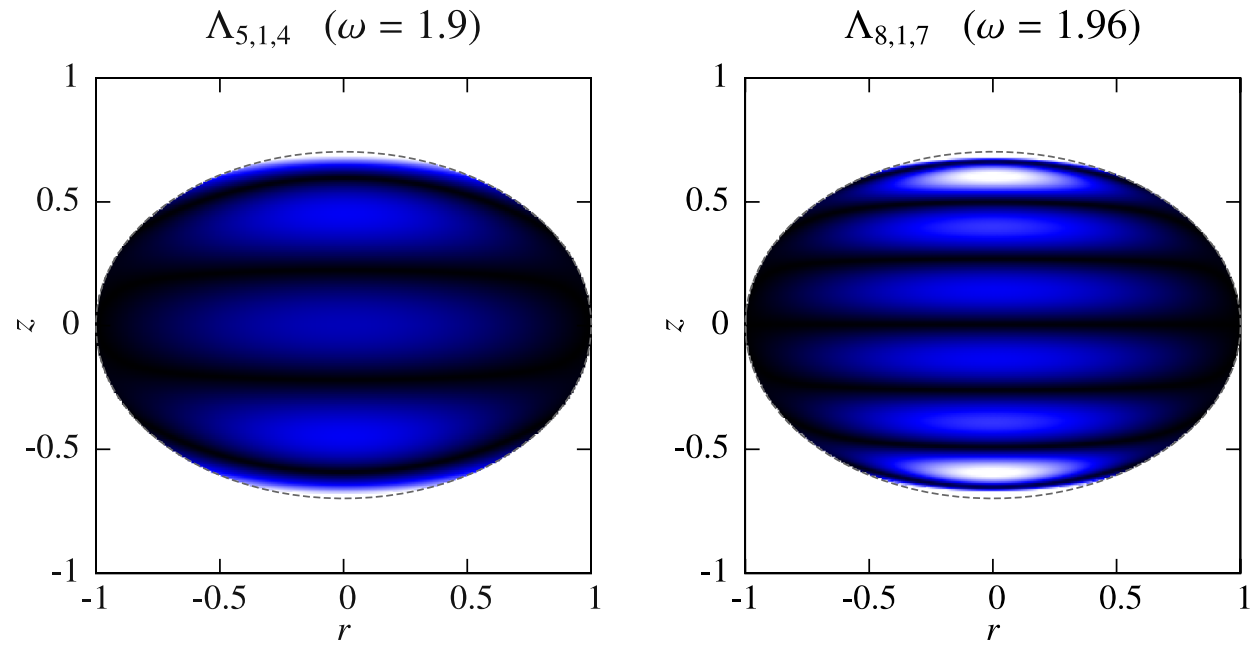

FIG. 6. Amplitude $|\boldsymbol{Q}|$, as defined by Eq. (2.30) in Kerswell, ${ }^{35}$ of an inertial mode $\Lambda_{n, m, K}$ for a spheroid with $c=0.7$. Bright and dark colors correspond to large and low amplitudes, respectively. The results are shown in a meridional slice and we compare two inertial modes with an azimuthal wave number $m=1$ and an eigenfrequency $\omega$ close to 2 .

at every time step, and we perform a spectral analysis of these signals. Since we use an adaptive time step method, the signals from the numerical simulations need to be evaluated on a uniform grid which is done using a sixth order Lagrangian interpolation scheme. For each of the three velocity components, we compute the discrete Fourier transform of the signal during an arbitrary time interval. Note that the input signal is not necessarily periodic so that we need to multiply it by a periodic window function to avoid spectral leakage associated with the discontinuity between the start and the end of the signal. Here, we use the Hanning window defined as

$$
w(n)=\frac{1}{2}\left[1-\cos \left(2 \pi \frac{n}{N-1}\right)\right], 0 \leq n \leq N-1,
$$

where $N$ is the total number of samples. We checked that using other window functions does not qualitatively change the results.

Figure 7(a) shows the resulting power spectra for cases $A 1, A 2$, and $A 4$, where we only used the signals obtained after the instability has saturated. For case $A 1$, the flow is actually stable so that we only observe two peaks, one corresponding to the base flow at $\omega=f=4$ and one corresponding to the zonal flow at $\omega=0$. Case $A 2$ is unstable and the peaks associated with the base and zonal flows are still present and unchanged, but we now see a peak at $\omega=f / 2=2$ and subsequent harmonics at $\omega=6,8, \ldots$. We argue that these peaks are associated with the two resonating inertial modes at the
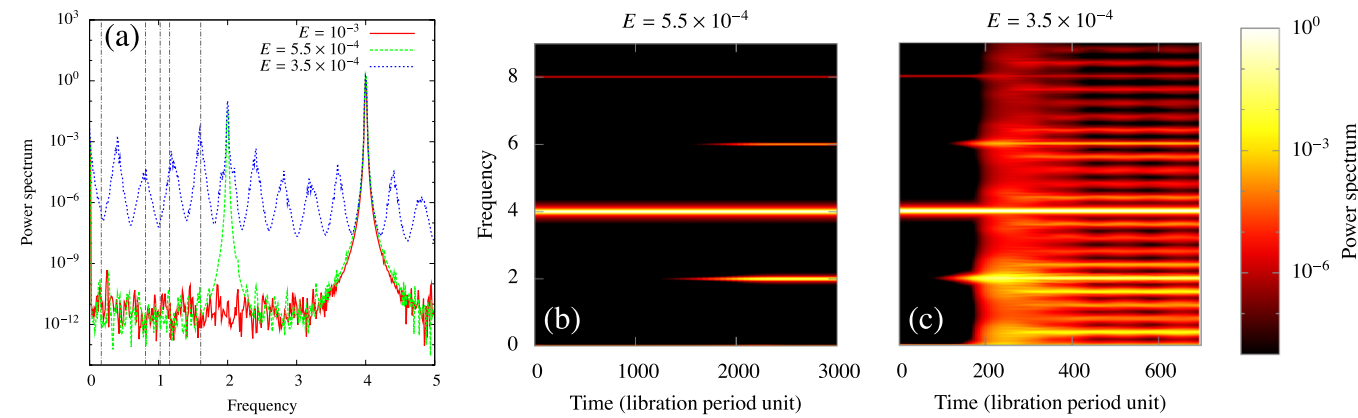

FIG. 7. Temporal power spectrum for cases $A 1\left(E=10^{-3}\right), A 2\left(E=5.5 \times 10^{-4}\right)$, and $A 4\left(E=3.5 \times 10^{-4}\right)$. (a) Power spectra are averaged over 500 libration periods during the saturated phase. The vertical dotted-dashed lines correspond to the theoretical predictions of Vantieghem ${ }^{36}$ for the eigenfrequencies of linear and quadratic inertial modes in the ellipsoid. Time evolution of the power spectrum with a sliding window of 50 libration periods for cases $A 2$ (b) and $A 4$ (c). 
origin of the elliptical instability with frequencies $|\omega| \lesssim 2$ which satisfied the resonance condition $\left|\omega_{2}-\omega_{1}\right| \approx f$. Finally, the third spectrum corresponds to the case $A 4$, where the Ekman number is reduced even further to $3.5 \times 10^{-4}$. In addition to the previous features, many additional peaks are now clearly visible. A first indication that these are also inertial modes is that their frequencies are comparable with eigenfrequencies of linear and quadratic inertial modes of the full ellipsoid ${ }^{36}$ as shown in Figure 7(a) (additional theoretical frequencies would be found by increasing the order of the polynomial expansion). These additional frequencies we observed are, in decreasing order of amplitude, $\omega \approx 1.6,0.4,1.2$, and 0.8 . These frequencies are therefore compatible with quadratic interactions between the primary inertial modes at $\omega \approx 2$ and two of the daughter modes (i.e., 1.6 and 0.4 on the one hand, 1.2 and 0.8 on the other hand), as further discussed below. Note that for the particular simulations $A 2-A 4$ considered here, the saturation of the elliptical instability does not lead to turbulence, as the Ekman number is too large. The power spectrum remains quasi-discrete since mode couplings are very limited for these viscously dominated cases. The two right panels in Figure 7 show the time evolution of the power spectra for cases $A 2$ and $A 4$. At each time step, the Fourier analysis is performed over a window of 50 libration periods. The chronology of the various resonances becomes clear. The libration forcing sustains the base flow at $f=4$ from the beginning of the simulation. We then observe a primary resonance involving two inertial modes with frequencies $\omega \approx \pm 2$ and the base flow at $\omega=4$. As the amplitude of these primary inertial modes becomes larger, and if the Ekman number is low enough (i.e., $E \leq 4 \times 10^{-4}$ in our case), secondary quadratic interactions are allowed with two sets of daughter inertial modes whose frequencies are given by the resonance condition $\left|\omega_{2}-\omega_{1}\right|=2$. Note that no further resonances are observed for case $A 4$.

We now compare numerical and experimental data in order to confirm the quadratic interactions observed numerically in the laminar regime. In particular, we focus on simulation $A 6$, for which $E=10^{-4}$, whereas we recall that $E=2 \times 10^{-5}$ for the experiment. Since the Ekman numbers are different, we expect quantitative differences between the two approaches. Note that both simulation $A 6$ and the experiment are in the turbulent regime, but as we show below, we can still distinguish between the dominant low-frequency interactions and the background turbulence noise. The frame rate of the camera used for the PIV is 23.9 frames per second. In our dimensionless units, this leads to a maximum frequency of $\omega=24$ which is not enough to compare with the high-frequency range available numerically. We note in addition that experimental time spectra tend to be flat at high-frequencies, probably due to uncertainties. For these reasons, we focus on the low frequencies $\omega \leq 4$. The behaviors of the high frequencies in the numerical simulations will be discussed in Sec. V E. Numerical probes are placed at the same location as for the PIV measurements and the power spectra are computed using a sliding window of 20 libration periods. Experimental
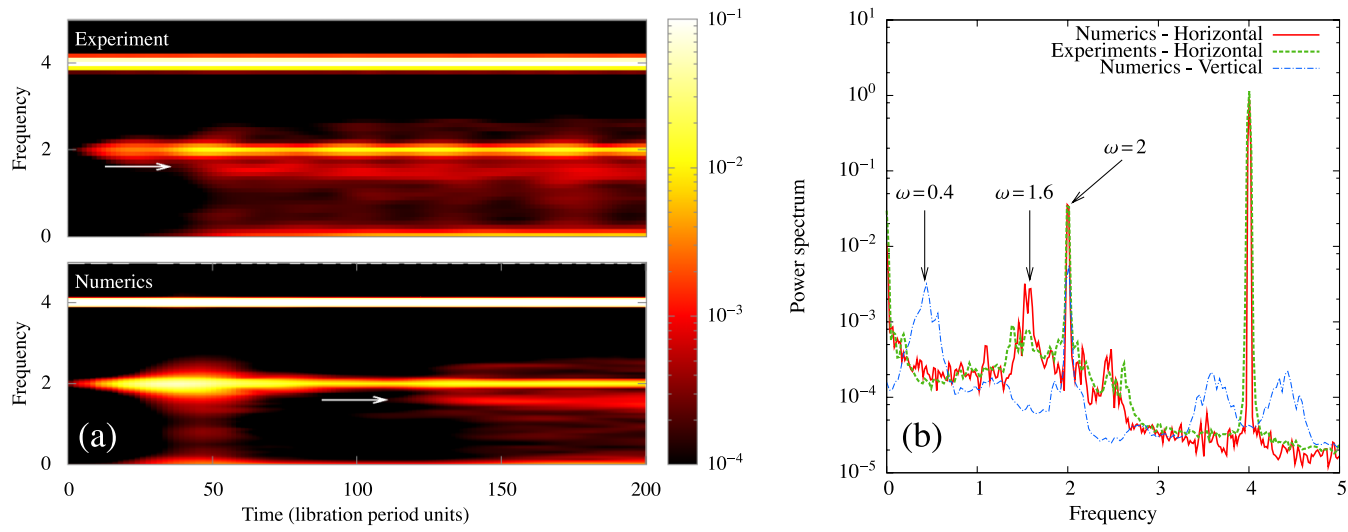

FIG. 8. Comparison between experimental and numerical results. (a) Spectrograms computed using horizontal velocity signals in the equatorial plane only. The top panel corresponds to the experimental results at $E=2 \times 10^{-5}$ (case $V$ in Grannan et al. ${ }^{22}$ ) whereas the bottom panel corresponds to the numerical case $A 6$ at $E=10^{-4}$. The white arrow indicates the appearance of the particular frequency $\omega=1.6$ involved in the secondary quadratic interactions. (b) Power spectra time-averaged during the saturated phase. We also show the numerical power spectrum associated with the vertical component (not available experimentally). 
and numerical results are presented in Figure 8(a), where we show the spectrogram associated with the equatorial horizontal flow only. In both cases, we see the dominant contribution from the base flow at $\omega=4$ from the beginning of the experiment. As time is evolving, the first resonance with the primordial inertial modes at $\omega=2$ occurs as already observed in the laminar regime. Once the primary inertial modes have grown in amplitude, the secondary resonance at $\omega=1.6$ is also visible in both cases and is indicated by a white arrow. This secondary resonance occurs earlier in the experiment than in the numerical simulation due to the lower Ekman number in this case (and therefore a larger growth rate for the elliptical instability).

Interestingly, the frequency $\omega=0.4$, already observed numerically in Figure 7, is not seen in the horizontal flow. However, when looking at the vertical component from the numerics in the same equatorial plane, we recover the frequency $\omega=0.4$ required by the resonance condition. This is visible in Figure 8(b), where we show the power spectra averaged during the saturated phase for the numerical simulation (the vertical component of the flow is not available experimentally). We also show in Figure 8(b) the horizontal power spectra from both the experiment and the numerics. Note the excellent agreement between both approaches in this frequency range. No rescaling has been applied.

In order to extract the spatial structure of the different components of the flow, we follow the approach used by several authors ${ }^{39-41}$ in the context of internal gravity or inertial wave attractors. The velocity field is filtered at a particular frequency $\omega_{f}$ according to

$$
\hat{\boldsymbol{u}}\left(\omega_{f}, \boldsymbol{x}\right)=\frac{\omega_{f}}{N \pi} \int_{t_{i}}^{t_{f}} \boldsymbol{u}(\boldsymbol{x}) e^{i \omega_{f}\left(t-t_{i}\right)} \mathrm{d} t,
$$

where the arbitrary times $t_{i}$ and $t_{f}$ are separated by $N$ periods $T=2 \pi / \omega_{f}$. Here, we consider the velocity fields filtered at $\omega_{f}=f, \omega_{f}=0$, and $\omega_{f}=f / 2$, which correspond, respectively, to the base flow, the zonal flow, and the primordial inertial modes. Figure 9 shows the amplitude of each of these filtered velocity fields. Base flow (6) is recovered in the bulk. The zonal flow is averaged over the initial phase before the instability develops. We recover the strong prograde circulation close to the equatorial boundary and the weaker retrograde interior jet, as already discussed in Sec. V C. Finally, the inertial modes correspond to layered structures dominated by horizontal motions and are very similar to the inertial modes for a spheroid as shown in Figure 6. In particular, the structure observed in the rightmost panel of Figure 9 corresponds to the inertial mode $\Lambda_{7,1,6}$ with an eigenfrequency of $\omega=1.95$. The wave number $n$ of the inertial modes observed by this filtering approach increases as we decrease the Ekman number. We indeed observe the inertial mode $\Lambda_{8,1,7}$ for cases $A 6$ and $A 7$, which is consistent with the experimental observation made by Grannan et $a .^{22}$ Although removed from Figure 9 by the averaging process defined in Eq. (20), the inertial modes also have an azimuthal wave number $m=1$ component, as expected due to the resonance conditions with the elliptical base flow with $m=2$.

Although the appearance of a mode at half the frequency of the forcing is a strong indication of a parametric resonance, the power spectrum is not enough to conclude since the phase information is lost. In order to explicitly show that quadratic couplings are responsible for the growth of the $\omega \approx 2$ modes, one has to rely on higher-order spectral analysis. In particular, we choose the consider
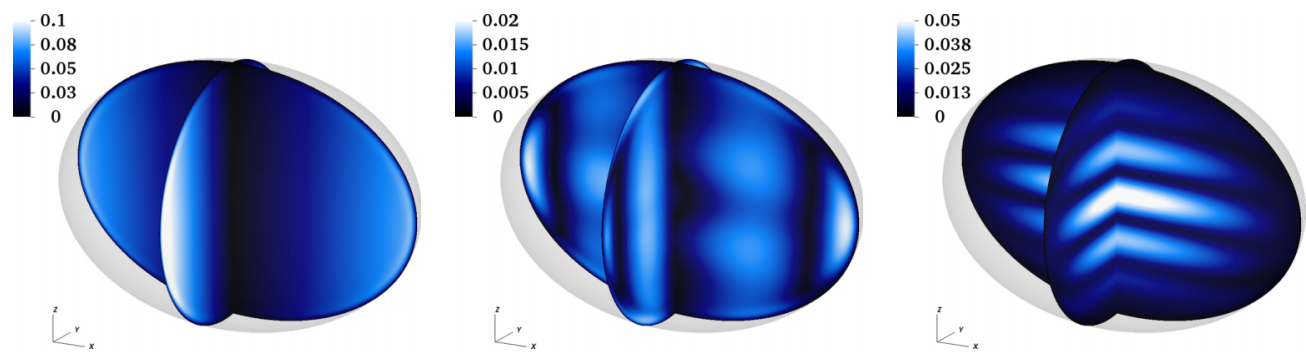

FIG. 9. Velocity and amplitude associated with filtered velocity fields at various frequencies using Eq. (20) for case $A 3$ $\left(E=5 \times 10^{-4}\right)$. Left: Base flow obtained by filtering at $\omega_{f}=f$ over 100 libration periods. Middle: Zonal flow obtained by time averaging. Right: Inertial modes filtered at $\omega_{f}=f / 2$. 
the bicoherence defined as

$$
B^{2}\left(f_{1}, f_{2}\right)=\frac{\left|\sum_{i=1}^{N} u_{i}\left(f_{1}\right) u_{i}\left(f_{2}\right) u_{i}^{*}\left(f_{1}+f_{2}\right)\right|^{2}}{\sum_{i=1}^{N}\left|u_{i}\left(f_{1}\right) u_{i}\left(f_{2}\right)\right|^{2} \sum_{i=1}^{N}\left|u_{i}\left(f_{1}+f_{2}\right)\right|^{2}},
$$

where $u_{i}\left(f_{1}\right)$ is the temporal Fourier mode of the velocity component $i$ at frequency $f_{1}$ and the star denotes the complex conjugation. Time signals are split into $N$ individual time windows over which the Fourier components are calculated using the same approach as for the power spectrum discussed previously. As $N$ increases, the bicoherence $B^{2}\left(f_{1}, f_{2}\right)$ tends to zero if the amplitude of the frequencies $f_{1}, f_{2}$, and $f_{1}+f_{2}$ are zero, or if the phase of each of these frequencies is uncorrelated. Values of the bicoherence close to unity indicate phase coupling, where the signal phases $\phi_{1}, \phi_{2}$, and $\phi_{3}$ at frequencies $f_{1}, f_{2}$, and $f_{3}=f_{1}+f_{2}$, respectively, follow the relation $\phi_{3}=\phi_{1}+\phi_{2}$. We show in Figure 10 the bicoherence map for cases $A 2$ and $A 4$. The results are averaged over 50 different probe signals and over all three components of the velocity. For case $A 2$ where only the inertial modes $\omega=2$ are excited, a clear peak in the bicoherence is seen for $f_{1}=f_{2}=2$ indicating coherent phases between modes at those frequencies. As the Ekman number decreases, the other modes already discussed in Figure 7 are also phase coherent, as indicated by large values of the bicoherence. This further confirms that the observed frequencies $\omega<2$ are generated by quadratic interactions.

This small window of parameters where the primary elliptical instability saturates in a laminar state is only obtained for $6 \times 10^{-4}<E<3 \times 10^{-4}$. This is consistent with the experimental results of Eloy et al. $^{42}$ who observed a laminar saturation of the primary elliptical instability for $E>2.5 \times 10^{-4}$ and with the theoretical results of Kerswell ${ }^{43}$ who predicted that the inertial mode should become linearly unstable to triadic interactions at $E \leq 2.5 \times 10^{-4}$. In our case, smaller values of the Ekman number do not lead to additional resonances but to small-scale disorder. Note that the detailed mechanism by which this transition to turbulence initially occurs remains to be identified. We focus in Sec. V E on the statistical properties of the developed turbulent state.

\section{E. Fully developed turbulent regime}

We now focus our attention on simulations leading to a turbulent saturated regime: cases $A 5-A 7$. In all turbulent cases, the flow is far from being homogeneous due to the presence of solid boundaries but also due to energy injection mechanism by the sudden breaking of inertial modes. To identify the spatial region where turbulence is preferentially driven, we compute the spatial distribution of the turbulent kinetic energy as follows:

$$
\mathcal{K}=\frac{1}{2}\left\langle\left(\boldsymbol{u}^{\prime}-\left\langle\boldsymbol{u}^{\prime}\right\rangle\right)^{2}\right\rangle,
$$

where $\boldsymbol{u}^{\prime}=\boldsymbol{u}-\boldsymbol{U}_{b}$ is the fluctuating velocity around the base flow given by Eq. (6). The brackets denote here a temporal average performed over 100 libration periods during the quasi-steady phase.
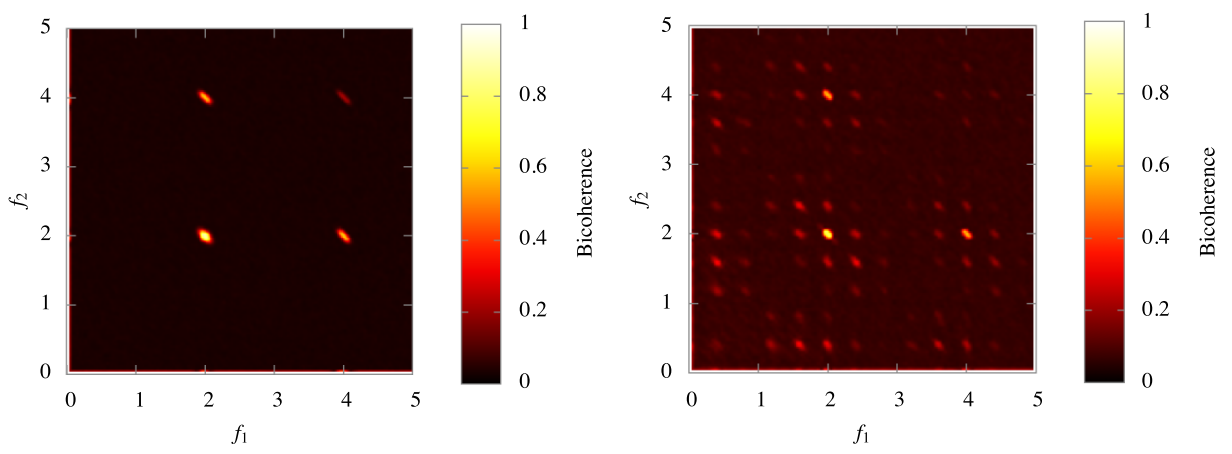

FIG. 10. Bicoherence as defined by Eq. (21) for cases $A 2$ (left) and $A 4$ (right). Values close to unity indicate a near-perfect phase coherence between energetic modes at frequencies $f_{1}, f_{2}$, and $f_{1}+f_{2}$. 

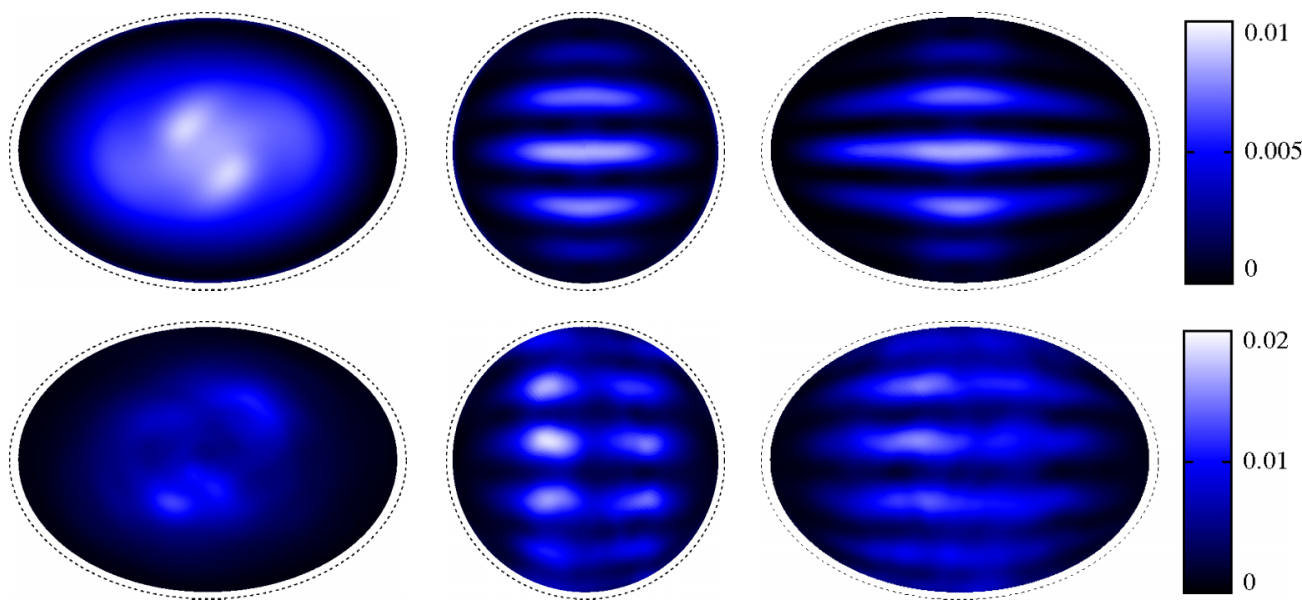

FIG. 11. Turbulent kinetic energy $\mathcal{K}$ as defined by Eq. (22) plotted on the $x y$ equatorial plane (left), the $y z$ meridional plane (middle), and the $x z$ meridional plane (right). Results at the top correspond to the laminar case $A 4$ whereas results at the bottom correspond to the turbulent case $A 6$. In both cases, the average required in Eq. (22) is performed over approximately 100 libration periods.

A similar approach has been used to characterize the turbulence driven in a precessing sphere. ${ }^{44}$ The spatial distribution of the turbulent kinetic energy is shown in Figure 11 for cases $A 4$ (top row) and $A 6$ (bottom row). Case $A 4$ is still laminar and is shown for reference. In that case, the fluctuating flow is dominated by the primordial inertial modes $\Lambda_{7,1,6}$, and their signature is clearly visible in the fluctuating kinetic energy (compare with Figures 6 and 9, for example). Interestingly, the overall layered structure of the inertial modes is also visible in the turbulent kinetic energy distribution associated with the fully turbulent case $A 6$. Note that the average process used in Eq. (22) converges after approximately 20 libration periods for case $A 4$, whereas case $A 6$ requires averaging over more than 100 libration periods. The maximum of the turbulent kinetic energy in the turbulent case occurs when the amplitude of the inertial modes is maximum. This correlation between the structure of the inertial modes and the turbulent kinetic energy indicates that the primordial inertial modes are still being excited even after the instability saturates and remains of larger amplitude than the small-scale turbulent flow. Even if turbulence is filling most of the ellipsoid, only motions generated at the maximum of the inertial modes' amplitudes are long-lived and continuously fed by the instability whereas other fluctuating motions cascade to small-scales and are rapidly dissipated by viscosity.

We now repeat the same spectral analysis as in Sec. V D, in order to extract the temporal power spectra in the turbulent regime. The time signals are multiplied by a Hanning window over 200 libration periods and we average the results over 100 different probes located within the bulk of the ellipsoid. Figure 12(a) shows the corresponding spectra in $\log -\log$ scale, where we also plot the results corresponding to cases $A 2$ and $A 4$, already discussed in Sec. V D. It is clear that, for $E \leq 2 \times 10^{-4}$, the spectra are now continuous with a large range of excited frequencies. The zonal flow (not visible in this logarithmic scale), the base flow, and primordial inertial modes are still clearly distinguishable and dominate the spectra for all Ekman numbers considered. The secondary quadratic interactions involving $\omega=0.4$ and $\omega=1.6$ are also still observable whereas the frequencies $\omega=0.8$ and $\omega=1.2$ are now dominated by low-frequency components with a rather flat spectrum. As the Ekman number decreases, the high-frequency part of the spectrum is more and more populated, which further confirms that the flow is in a developed and sustained state of turbulence, with small spatial scales and short time scales. The dashed-dotted line shown in Figure 12(a) corresponds to a slope $\omega^{-3}$ and is shown for reference. Note that from an energetic point of view, the sustained turbulent regime is still dominated by the base flow, followed by the primary inertial modes (which is consistent with the result presented in Figure 11), the secondary inertial modes, and finally the small-scale turbulent flow. 

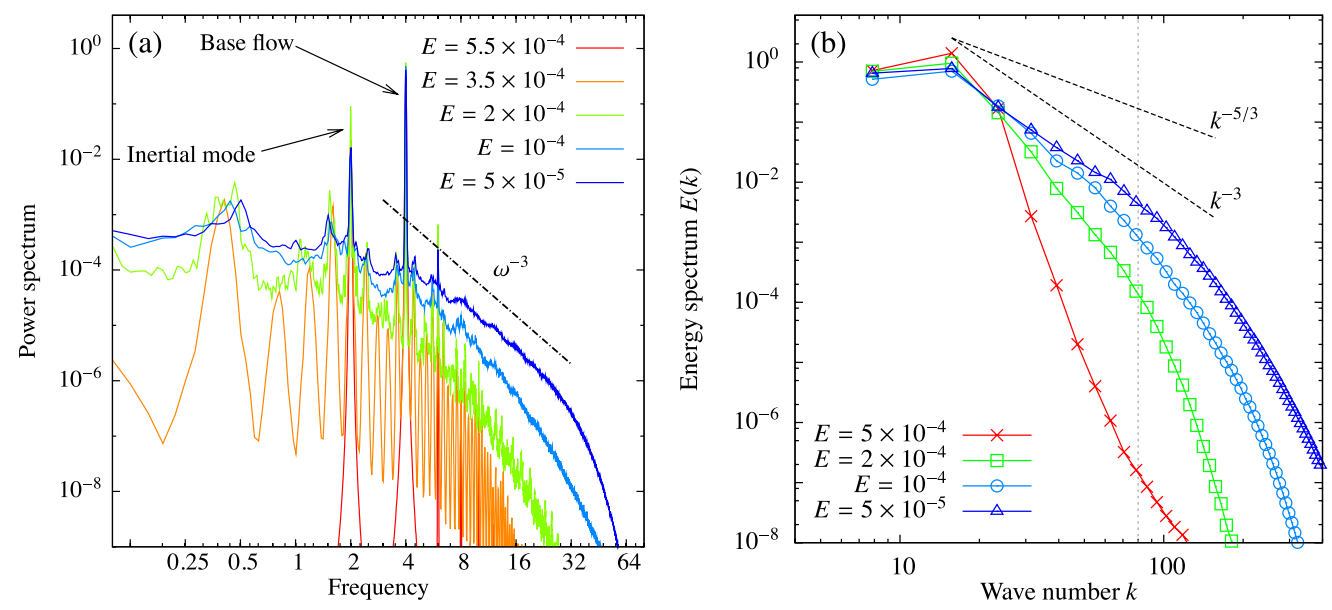

FIG. 12. (a) Temporal power spectrum averaged over 100 probes distributed within the ellipsoid. The temporal spectral analysis is performed after the instability has saturated. The dashed-dotted line corresponds to the power law $\omega^{-3}$ and is shown for reference. (b) Spatial power spectrum computed inside a cube centered around the origin. Velocity signals are made spatially periodic by using window function (19) in all three directions and the resulting energy spectra are time-averaged during the saturated phase. The vertical dashed line indicates the Zeman scale as defined in the main text.

Additional information can be obtained by looking at the energy distribution among the different spatial scales of the flow. As discussed above, the turbulent flow is inhomogeneous but we nevertheless use a Fourier decomposition. We focus on a cube centered around the origin and with side 0.8 , fully enclosed inside the ellipsoid and outside of the boundary layers. The velocity components are interpolated with spectral accuracy on a uniform Cartesian grid and the periodicity is enforced using window function (19) in all three directions. The energy spectrum is then computed as

$$
E_{K}(k)=\sum_{k-1 / 2<|k|<k+1 / 2} \hat{\boldsymbol{u}}(\boldsymbol{k}) \cdot \hat{\boldsymbol{u}}^{*}(\boldsymbol{k}),
$$

where $\hat{\boldsymbol{u}}(\boldsymbol{k})$ is the three-dimensional Fourier transform of $\boldsymbol{u}(\boldsymbol{x})$ and the star denotes complex conjugation. The resulting spatial energy spectra are shown in Figure 12(b), where the results are further averaged over time during the saturated phase. As the Ekman number decreases, small spatial scales appear and a tendency toward a scaling $E(k) \propto k^{-3}$ is observed. Such a scaling for the energy spectrum has also been observed in homogeneous simulations of the elliptical instability using a shearing-box approximation and a tidal forcing. ${ }^{45}$ We recall that the small-scale Rossby number is below unity for all our simulations (see Table I). The exponent observed in our temporal and spatial energy spectra could therefore be related to the effect of the background rotation on the small-scale turbulence generated by the inertial mode breaking. In the case of homogeneous rotating turbulence, a transition from the usual $-5 / 3$ scaling of Kolmogorov theory to the steeper -3 scaling is associated with a reduction of the forward energy cascade due to Coriolis effects. ${ }^{46}$ Note however that our Rossby number is close to unity ( $R o \approx 0.5$ for case $A 7$, for example). We therefore expect the Kolmogorov scaling to reappear at small scales as the effective Rossby number increases. The reisotropization of rotating turbulence is usually associated with the so-called Zeman scale ${ }^{47,48}$ defined as $l_{\Omega}=\sqrt{\epsilon_{t} / \Omega_{0}^{3}}$, where $\epsilon_{t}$ is the kinetic energy dissipation rate and $\Omega_{0}$, the rotation rate. Using the bulk dissipation rate from case $A 7$, the critical wave number associated with this Zeman scale is $k_{\Omega} \approx 80$, which corresponds to the end of the inertial range as indicated by the vertical dashed line in Figure 12(b). It is therefore possible that for even lower Ekman numbers, we would observe an isotropic state of turbulence at scales smaller than $l_{\Omega}$, as it has already been reported in the case of homogeneous rotating turbulence. ${ }^{49,50}$ We cannot verify this claim at this stage since we are limited in the range of spatial scales we can consider, having to solve for the large-scale elliptical base flow responsible for the instability in addition to the small-scale turbulent flow. 


\section{F. Reducing the eccentricity}

In order to compensate for the dissipation in the viscous boundary layers, we have to consider sufficiently large values of the deformation $\beta$ for the elliptical instability to be numerically tractable (i.e., for the growth rate to be large enough). So far, the value we considered was $\beta=0.34$, which was chosen to be the same as in the laboratory experiment of Grannan et al. ${ }^{22}$ However, the eccentricity of celestial objects is usually much smaller ${ }^{10,20}$ so that decreasing $\beta$ and studying its effect on the resulting flow are of interest.

In this section, we repeat simulation $A 6$, but with a reduced equatorial deformation of $\beta=0.17$ and $\beta=0.26$ (cases $B 1$ and $B 2$, respectively, in Table I). For all cases, the aspect ratio $c / b=1$ and the Ekman number $E=10^{-4}$ are kept constant. By reducing $\beta$, we reduce the super-criticality and, thus the growth rate $\sigma$ of the instability (see the growth rate defined by Eq. (10)). This means that our simulation is getting closer and closer to the onset of the elliptical instability. One would like to keep reducing $E$ as $\beta$ is decreased in order to keep the ratio $E / E_{c}$ constant, but this is unfortunately not numerically feasible with our current computing capabilities.

The time evolution of the volume averaged vertical kinetic energy is shown in Figure 13 for the three cases $\beta=0.17,0.26$, and 0.34 . As expected, the growth rate of the instability is reduced as $\beta$ is decreasing. In addition, the final amplitude at which the instability saturates is again decreasing as $\beta$ decreases. The main difference between the three cases lies in the transient phase before the eventual quasi-steady saturation. As already observed in Sec. V A, for $\beta=0.34$, the laminar state is never recovered after the instability grows. The first saturation does lead to a decay in the vertical kinetic energy for a short time, but another growth eventually takes place and a quasi-steady state is reached after a couple of cycles. This is at odds with the case $\beta=0.17$, where a complete relaminarisation of the flow is observed after the first development of the instability (around $t \approx 500$ in Figure 13, for example). In addition, many cycles of growth and collapse are required to eventually reach a quasi-steady phase. The saturated phase is still moderately turbulent, but the small-scale Reynolds is much smaller than for $\beta=0.34$. The case $\beta=0.26$ is somewhere in between with alternation between intermittent phases (for $t \approx 300$ or $t \approx 700$, for example) and sustained phases (for $t \approx 500$ ). Note that in all cases, we still observe the same resonance mechanism (primordial inertial modes excited by the elliptical instability followed by subsequent triadic interactions) although the frequencies of the modes involved are slightly shifted due to the change in geometry. As in Figures 7, 8, and 12, the inertial modes are still dominating the spectrum in the frequency range $0<\omega<2$. This is, however, only true for our cases in which $E$ is fixed and $\beta$ is varied. It might not be applicable to the geophysically relevant regime where both $E$ and $\beta$ are reduced simultaneously in order to remain far from the instability threshold.

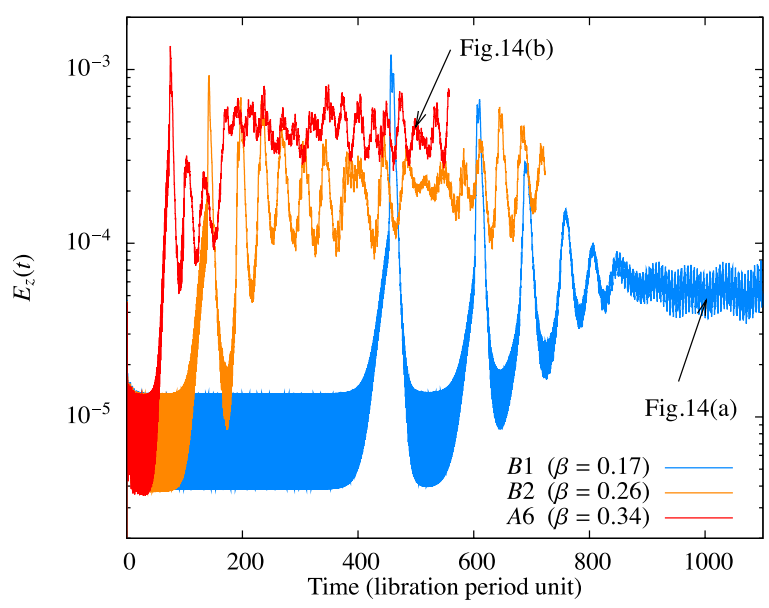

FIG. 13. Volume averaged vertical kinetic energy for three different ellipticities of the container (cases $B 1, B 2$, and $A 6$ ). Apart from $\beta$, the parameters are the same for all three cases. The arrows indicate the times at which the visualizations shown in Figure 14 are realized. 
This transition between a sustained level of turbulence and an intermittent regime has already been observed in shearing-box simulations of the elliptical instability in a tidal forcing context. ${ }^{45}$ In that paper, the authors found a critical value for the eccentricity of the streamlines of $\beta \approx 0.15$ above which a sustained level of turbulence activity is observed. While our setup is different due to the presence of solid boundary conditions and the use of a librational forcing to excite the elliptical instability, the transition observed in our simulations might be of similar nature. We indeed observe a very long intermittent transient when $\beta=0.17$, so that it is possible that an even lower value of the equatorial deformation would only lead to an intermittent behavior. However, due to the stabilizing effect of viscous dissipation, it is not possible to further reduce $\beta$ while keeping the growth rate sufficiently large for the instability to be numerically tractable.

Barker and Lithwick ${ }^{45}$ argued that the presence of large-scale vortices invariant in the vertical direction is responsible for the decay observed after the initial collapse. This columnar flow is produced by the decay of the small-scale turbulence under the influence of rotation and could damp the resonances required to sustain the small-scale turbulence. There are indeed indications that such a vertically invariant flow does develop in our simulations as $\beta$ is decreased, as can be seen in Figure 14 , where we show the vertical velocity at an arbitrary time during the saturated phase in a meridional plane for both $\beta=0.17$ and $\beta=0.34$. We observe vertically coherent structures for the case with $\beta=0.17$ whereas the flow appears to be more isotropic when $\beta=0.34$. In addition, reducing $\beta$ is also dramatically increasing the importance of viscous effects as can be seen in Figure 13 where the growth rate rapidly decreases with $\beta$. A detailed analysis of the interaction between these large-scale coherent structures and the inertial modes excited by the elliptical instability is beyond the scope of this paper. It therefore remains to be seen whether the elliptical instability saturates in an intermittent or quasi-steady manner in the geophysical regime at low- $\beta$ and low- $E$. This is a crucial question since the efficiency of the elliptical instability in terms of energy dissipation and dynamo action is at play.

\section{G. Varying the librating frequency}

In this section, we briefly discuss the behavior of the system for another librating frequency $f=2.4$. It was shown experimentally by Grannan et $a l .{ }^{22}$ that varying the librating frequency leads to a variety of behaviors as the resonant modes change. One of the reasons why we focus on the $f=4$ case so far is that it is very easy to obtain a turbulent regime numerically since the critical Ekman number for instability is quite large. This might be related to the peculiar spatial structure of the inertial modes with $|\omega| \lesssim 2$, as shown in Figure 6. As the librating frequency decreases, turbulence is only observed for more extreme values of the control parameters. At the fixed experimental Ekman number of $E=2 \times 10^{-5}$, the critical libration amplitude $\epsilon$ to reach a turbulence regime is $\epsilon \approx 0.12$ for $f=4$, whereas it is $\epsilon \approx 0.7$ for $f=1.46$ (see Figure 5 in Grannan et al. ${ }^{22}$ ). For these reasons, numerical simulations of the libration-driven elliptical instability at lower libration frequencies are only turbulent for large values of the libration amplitude. This leads to numerical complications as the boundary layers can then be unstable to centrifugal instabilities, eventually leading to turbulence before the elliptical instability develops.
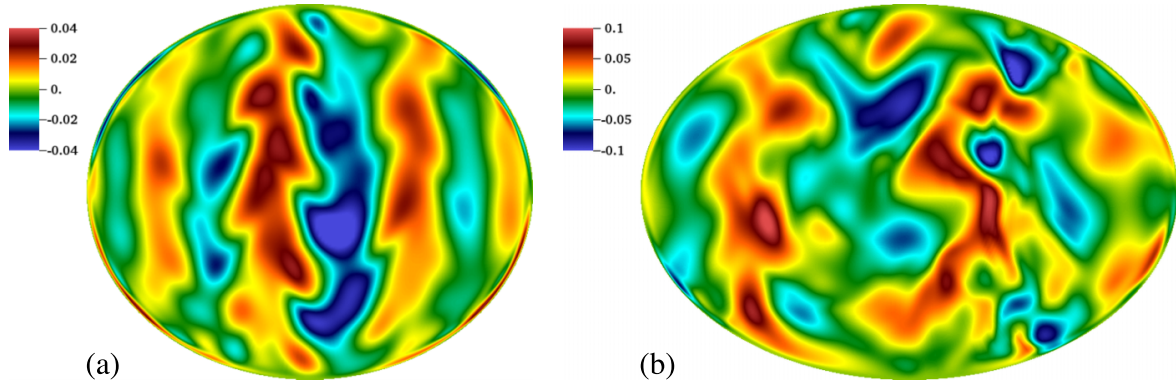

FIG. 14. Vertical component of the velocity in the $(x, z)$ meridional plane during the saturated phase for (a) $\beta=0.17$ $(t \approx 1000)$ and (b) $\beta=0.34(t \approx 500)$, as indicated by arrows in Figure 13. 

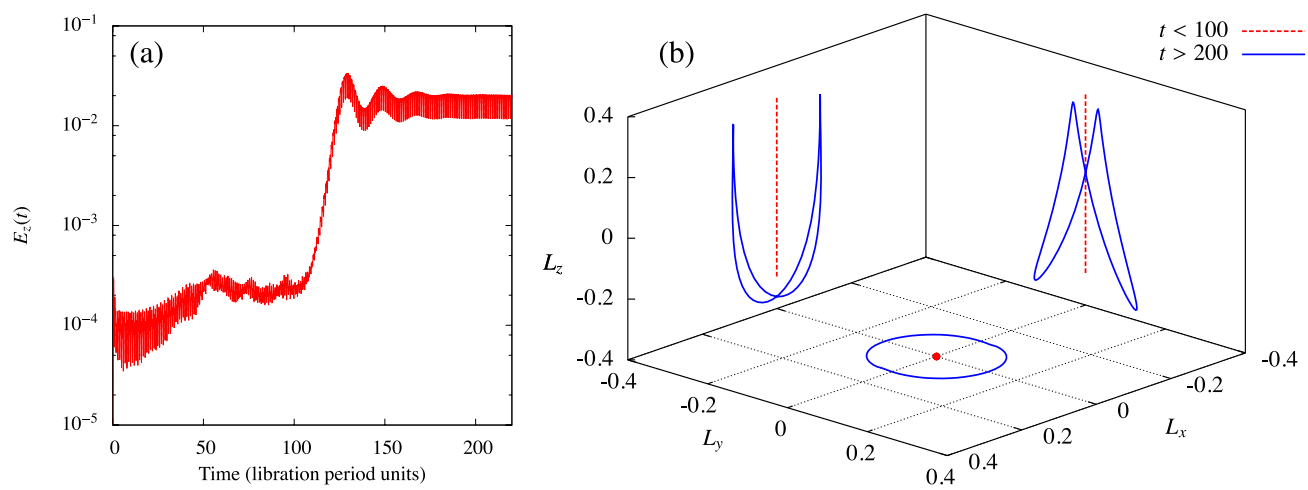

FIG. 15. Results for case $C 1$ with $f=2.4, \epsilon=1.2$, and $E=10^{-4}$. (a) Time evolution of the vertical kinetic energy. (b) Phase diagram of the three components of the angular momentum. We show the two-dimensional projections $\left(L_{x}(t), L_{y}(t)\right)$, $\left(L_{x}(t), L_{z}(t)\right)$, and $\left(L_{y}(t), L_{z}(t)\right)$, on each side.

As an example of other flows driven by the saturation of the elliptical instability, we consider the case $f=2.4, \epsilon=1.2, \beta=0.34$, and $E=10^{-4}$. Figure 15 (a) shows the time evolution of the squared vertical velocity. Contrary to the case $f=4$, the Ekman boundary layers do not remain laminar during the initial phase of the instability. Taylor-Görtler vortices are generated through a centrifugal instability in the equatorial boundary regions. ${ }^{11}$ This is a direct consequence of the large value of $\epsilon$ considered here. The fluctuations associated with these vortices are visible in Figure 15 (a) for $0<t<100$. The exponential growth phase is clearly visible for $100<t<150$ and is followed by the saturation of the instability. Note that the saturated phase corresponds to a quasi-steady evolution of the kinetic energy, contrary to the case with $f=4$, where a strongly fluctuating regime with low frequency modulations is observed at $E=10^{-4}$. The flow is not turbulent (as can be seen on the power spectrum or by direct visualization, not shown), but dominated by a large-scale periodic flow. The amplitude of this large-scale flow is much larger than the typical turbulent fluctuations observed for $f=4$ (see the values of $U_{\text {rms }}$ in Table I or the typical amplitude of $E_{z}$ in Figures 15(a) and 2(a)).

The main difference with the case $f=4$ is the angular momentum evolution. For $f=4$, the angular momentum remains purely vertical during the numerical experiment, even in the saturated quasi-steady phase. This is not the case for $f=2.4$ and the horizontal components of the angular momentum grow in amplitude when the instability starts to saturate. Figure 15(b) shows the phase diagram of the volume-averaged angular momentum defined as

$$
\boldsymbol{L}=\int_{V} \boldsymbol{x} \times \boldsymbol{u} \mathrm{d} V .
$$

Initially (i.e., for $t<100$ ), the Poincaré force generates a quasi-horizontal flow with dominant vertical angular momentum. As the instability develops, the horizontal components start to grow in amplitude while the periodic variations of the vertical component remain nearly unchanged. Finally, in the quasi-steady saturated phase, the direction of the angular momentum corresponds to the combination of a quasi-circular evolution of the horizontal angular momentum and the oscillating vertical component driven by the Poincaré force. The generation of a significant horizontal angular momentum is related to the excitation of the spin-over mode ${ }^{37,51}$ and is clearly visible when looking at the streamlines during the saturated phase, as shown in Figure 16. Note that such a large-scale flow does not remain laminar as the Ekman number decreases and it is indeed turbulent according to case $I V$ of Grannan et al..$^{22}$ for which $E=2 \times 10^{-5}$, but this turbulent regime is unfortunately not within reach of our numerical simulations. The amplitude of the excited flow crucially depends on the excitation of the spin-over mode and therefore on the libration frequency. When the spin-over mode is not excited (as in the case $f=4$ ), the amplitude of the fluctuations remains small when compared to the initial base flow, whereas they can become comparable when the spin-over mode is excited. More detailed studies about the excitation and saturation of the spin-over mode depending on the geometry, boundary conditions, and type of mechanical forcing are therefore needed. 


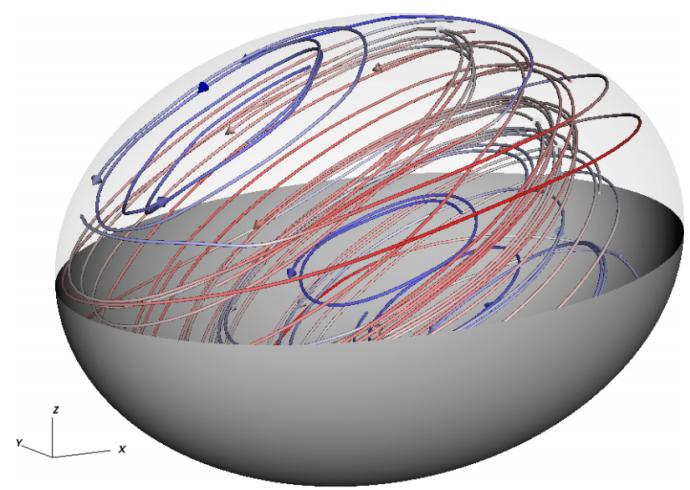

FIG. 16. Streamlines during the saturated phase colored with the flow speed for case $C 1$ in Table $\mathrm{I}$ with $f=2.4, \epsilon=1.2$, and $E=10^{-4}$.

\section{CONCLUSION}

We have qualitatively and quantitatively compared numerical and laboratory measurements of fluid motions inside a longitudinally librating rigid ellipsoid. In order to reduce the large number of control parameters, we have mostly focused on the interesting case $f=4$ for a fixed geometry $\beta=0.34$ and $c / b=1$, and for a fixed librating amplitude $\epsilon=0.8$. As the Ekman number is reduced, the elliptical instability is excited and we observe a transition from a laminar saturation to a fully developed sustained turbulent state. We observed both numerically and experimentally that the saturation of the elliptical instability drives an enhanced zonal flow whose amplitude increases as the Ekman number decreases. This is at odds with the zonal flow driven by nonlinearities in the Ekman boundary layers associated with the base flow only, which does not depend on the Ekman number and is therefore not relevant from a geophysical point of view.

The transition to turbulence is characterized by a succession of resonances between the dominant inertial modes at half the frequency of the forcing and the base flow, followed by additional triadic interactions between the dominant modes and other inertial modes of the ellipsoid. For the parameters considered, the turbulent saturation is characterized by an initial collapse of the primary inertial modes eventually leading to a sustained turbulent flow provided that the Ekman number is low enough (typically $E<3 \times 10^{-4}$ ). The resulting bulk turbulence is best characterized as rotating turbulence with a spatial and temporal energy spectra scaling approaching $k^{-3}$ and $\omega^{-3}$, respectively. The turbulence is however strongly inhomogeneous and is still being dominated by the primary inertial modes. As the eccentricity is reduced, a more intermittent regime is observed, with a complete relaminarization in some cases.

The current numerical and laboratory models are limited to large elliptical deformation and moderately low Ekman numbers. We consider cases which have centrifugally stable boundary layer flows in order to focus on the generation of bulk turbulence by the elliptical instability alone, but the interplay between these two instabilities could be of interest. ${ }^{52}$ We have focused our analyses on the particular case $f=4$, for which turbulence is more easily observed than for other librating frequencies and for which the spin-over mode is not excited. Finally, the fundamental difference between the sustained and intermittent turbulence regimes, and their respective relevance for planetary flows, needs to be addressed.

\section{ACKNOWLEDGMENTS}

We thank Adrian Barker and Paul Fischer for their help with the code Nek5000. This work was partly funded by the French Agence Nationale pour la Recherche (Grant Nos. ANR-14-CE33-0012 and ANR-13-JS05-0004-01). B.F. gratefully acknowledges financial support from the Programme National de Planétologie (PNP) of the Institut National des Sciences de l'Univers (INSU). B.F. would like to acknowledge support from IDRIS (Institut du Développement et des Ressources en Informatique Scientifique) for computational time on Turing (Project No. 100508) and from the 
HPC resources of Aix-Marseille Université financed by the project Equip@ Meso (No. ANR-10EQPX-29-01) of the program Investissements d'Avenir supervised by the Agence Nationale pour la Recherche (Project No. 15B011). AMG gratefully acknowledges the financial support of the Chateaubriand Fellowship Program for his 9-month stay in Marseille, France. AMG and JMA gratefully acknowledge financial support of the National Science Foundation (NSF) Geophysics Program and the NASA PG\&G (Planetary Geology and Geophysics) Program for funding the fabrication of the UCLA libration device. Part of this research was performed while the authors were visiting the Institute for Pure and Applied Mathematics (IPAM), which is supported by the NSF.

${ }^{1}$ C. A. Jones, "Planetary magnetic fields and fluid dynamos," Annu. Rev. Fluid Mech. 43, 583-614 (2011).

${ }^{2}$ M. Le Bars, M. A. Wieczorek, Ö. Karatekin, D. Cébron, and M. Laneuville, "An impact-driven dynamo for the early Moon," Nature 479, 215-218 (2011).

${ }^{3}$ C. A. Dwyer, D. J. Stevenson, and F. Nimmo, "A long-lived lunar dynamo driven by continuous mechanical stirring," Nature 479, 212-214 (2011).

${ }^{4}$ R. R. Kerswell and W. V. R. Malkus, “Tidal instability as the source for Io's magnetic signature,” Geophys. Res. Lett. 25, 603-606, doi:10.1029/98GL00237 (1998).

${ }^{5}$ M. Le Bars, D. Cébron, and P. Le Gal, "Flows driven by libration, precession, and tides," Annu. Rev. Fluid Mech. 47, 163-193 (2015).

${ }^{6}$ A. Tilgner, "Precession driven dynamos," Phys. Fluids 17(3), 034104 (2005).

${ }^{7}$ C.-C. Wu and P. H. Roberts, "On a dynamo driven topographically by longitudinal libration," Geophys. Astrophys. Fluid Dyn. 107, 20-44 (2013).

${ }^{8}$ D. Cébron and R. Hollerbach, “Tidally driven dynamos in a rotating sphere," Astrophys. J. 789, L25 (2014).

${ }^{9}$ R. L. Comstock and B. G. Bills, "A solar system survey of forced librations in longitude," J. Geophys. Res.: Planets 108, 5100, doi:10.1029/2003JE002100 (2003)

${ }^{10}$ J. Noir, F. Hemmerlin, J. Wicht, S. M. Baca, and J. M. Aurnou, "An experimental and numerical study of librationally driven flow in planetary cores and subsurface oceans," Phys. Earth Planet. Inter. 173, 141-152 (2009).

${ }^{11}$ M. A. Calkins, J. Noir, J. D. Eldredge, and J. M. Aurnou, "Axisymmetric simulations of libration-driven fluid dynamics in a spherical shell geometry," Phys. Fluids 22(8), 086602 (2010).

12 J. M. Lopez and F. Marques, "Instabilities and inertial waves generated in a librating cylinder," J. Fluid Mech. 687, 171-193 (2011).

${ }^{13}$ C.-Y. Wang, "Cylindrical tank of fluid oscillating about a state of steady rotation," J. Fluid Mech. 41, 581-592 (1970).

${ }^{14}$ A. Sauret, D. Cébron, C. Morize, and M. Le Bars, "Experimental and numerical study of mean zonal flows generated by librations of a rotating spherical cavity," J. Fluid Mech. 662, 260-268 (2010).

15 A. Sauret, D. Cébron, M. Le Bars, and S. Le Dizès, "Fluid flows in a librating cylinder," Phys. Fluids 24(2), 026603 (2012).

${ }^{16}$ J. Noir, D. Cébron, M. Le Bars, A. Sauret, and J. M. Aurnou, "Experimental study of libration-driven zonal flows in non-axisymmetric containers," Phys. Earth Planet. Inter. 204, 1-10 (2012).

${ }^{17}$ A. Sauret and S. Le Dizès, "Libration-induced mean flow in a spherical shell," J. Fluid Mech. 718, 181-209 (2013).

${ }^{18}$ K. Zhang, K. H. Chan, and X. Liao, "On fluid motion in librating ellipsoids with moderate equatorial eccentricity," J. Fluid Mech. 673, 468-479 (2011).

${ }^{19}$ K. H. Chan, X. Liao, and K. Zhang, "Simulations of fluid motion in ellipsoidal planetary cores driven by longitudinal libration,” Phys. Earth Planet. Inter. 187, 391-403 (2011).

${ }^{20}$ D. Cébron, M. Le Bars, C. Moutou, and P. Le Gal, "Elliptical instability in terrestrial planets and moons," Astron. Astrophys. 539, A78 (2012).

${ }^{21}$ D. Cébron, M. Le Bars, J. Noir, and J. M. Aurnou, “Libration driven elliptical instability,” Phys. Fluids 24(6), 061703 (2012).

${ }^{22}$ A. M. Grannan, M. Le Bars, D. Cébron, and J. M. Aurnou, "Experimental study of global-scale turbulence in a librating ellipsoid," Phys. Fluids 26(12), 126601 (2014).

${ }^{23}$ R. R. Kerswell, "Elliptical instability,” Annu. Rev. Fluid Mech. 34, 83-113 (2002).

${ }^{24}$ W. V. R. Malkus, "An experimental study of global instabilities due to the tidal (elliptical) distortion of a rotating elastic cylinder," Geophys. Astrophys. Fluid Dyn. 48, 123-134 (1989).

${ }^{25}$ T. S. Lundgren and N. N. Mansour, "Transition to turbulence in an elliptic vortex," J. Fluid Mech. 307, 43-62 (1996).

${ }^{26}$ N. Schaeffer and S. Le Dizès, "Nonlinear dynamics of the elliptic instability," J. Fluid Mech. 646, 471 (2010).

${ }^{27}$ D. M. Mason and R. R. Kerswell, "Nonlinear evolution of the elliptical instability: An example of inertial wave breakdown," J. Fluid Mech. 396, 73-108 (1999).

${ }^{28}$ M. O. Deville, P. F. Fischer, and E. H. Mund, High-Order Methods for Incompressible Fluid Flow (Cambridge University Press, 2002).

${ }^{29}$ P. F. Fischer, F. Loth, S. Lee, S. Lee, D. Smith, and H. Bassiouny, "Simulation of high-Reynolds number vascular flows," Comput. Methods Appl. Mech. Eng. 196, 3049-3060 (2007).

${ }^{30}$ B. Favier, A. J. Barker, C. Baruteau, and G. I. Ogilvie, "Non-linear evolution of tidally forced inertial waves in rotating fluid bodies," Mon. Not. R. Astron. Soc. 439, 845-860 (2014).

${ }^{31}$ J. Noir, M. A. Calkins, M. Lasbleis, J. Cantwell, and J. M. Aurnou, "Experimental study of libration-driven zonal flows in a straight cylinder," Phys. Earth Planet. Inter. 182, 98-106 (2010).

32 S. Le Dizès, "Three-dimensional instability of a multipolar vortex in a rotating flow," Phys. Fluids 12, 2762-2774 (2000).

${ }^{33}$ D. Cébron, S. Vantieghem, and W. Herreman, "Libration-driven multipolar instabilities," J. Fluid Mech. 739, 502-543 (2014).

${ }^{34}$ F. H. Busse, "Mean zonal flows generated by librations of a rotating spherical cavity," J. Fluid Mech. 650, 505 (2010). 
${ }^{35}$ R. R. Kerswell, “Tidal excitation of hydromagnetic waves and their damping in the Earth,” J. Fluid Mech. 274, 219-241 (1994).

${ }^{36}$ S. Vantieghem, "Inertial modes in a rotating triaxial ellipsoid," Proc. R. Soc. A 470(2168), 20140093 (2014).

${ }^{37}$ L. Lacaze, P. Le Gal, and S. Le Dizès, "Elliptical instability in a rotating spheroid," J. Fluid Mech. 505, 1-22 (2004).

${ }^{38}$ M. Le Bars, L. Lacaze, S. Le Dizès, P. Le Gal, and M. Rieutord, "Tidal instability in stellar and planetary binary systems," Phys. Earth Planet. Inter. 178, 48-55 (2010).

39 J. Hazewinkel, P. van Breevoort, S. B. Dalziel, and L. R. M. Maas, "Observations on the wavenumber spectrum and evolution of an internal wave attractor," J. Fluid Mech. 598, 373-382 (2008).

${ }^{40}$ N. Grisouard, C. Staquet, and I. Pairaud, "Numerical simulation of a two-dimensional internal wave attractor," J. Fluid Mech. 614, 1 (2008).

${ }^{41}$ L. Jouve and G. I. Ogilvie, "Direct numerical simulations of an inertial wave attractor in linear and nonlinear regimes," J. Fluid Mech. 745, 223-250 (2014).

${ }^{42}$ C. Eloy, P. Le Gal, and S. Le Dizès, "Experimental study of the multipolar vortex instability," Phys. Rev. Lett. 85, 3400 (2000).

${ }^{43}$ R. R. Kerswell, “Secondary instabilities in rapidly rotating fluids: Inertial wave breakdown,” J. Fluid Mech. 382, 283-306 (1999).

${ }^{44}$ S. Goto, A. Matsunaga, M. Fujiwara, M. Nishioka, S. Kida, M. Yamato, and S. Tsuda, "Turbulence driven by precession in spherical and slightly elongated spheroidal cavities," Phys. Fluids 26(5), 055107 (2014).

${ }^{45}$ A. J. Barker and Y. Lithwick, "Non-linear evolution of the tidal elliptical instability in gaseous planets and stars," Mon. Not. R. Astron. Soc. 435, 3614-3626 (2013).

${ }^{46}$ C. Cambon, N. N. Mansour, and F. S. Godeferd, "Energy transfer in rotating turbulence," J. Fluid Mech. 337, 303-332 (1997).

${ }^{47}$ O. Zeman, "A note on the spectra and decay of rotating homogeneous turbulence," Phys. Fluids 6, 3221-3223 (1994).

48 Y. Zhou, "A phenomenological treatment of rotating turbulence," Phys. Fluids 7, 2092-2094 (1995).

49 P. D. Mininni, D. Rosenberg, and A. Pouquet, "Isotropization at small scales of rotating helically driven turbulence," J. Fluid Mech. 699, 263-279 (2012).

${ }^{50}$ A. Delache, C. Cambon, and F. Godeferd, "Scale by scale anisotropy in freely decaying rotating turbulence," Phys. Fluids 26(2), 025104 (2014).

${ }^{51}$ D. Cébron, M. Le Bars, J. Leontini, P. Maubert, and P. Le Gal, "A systematic numerical study of the tidal instability in a rotating triaxial ellipsoid," Phys. Earth Planet. Inter. 182, 119-128 (2010).

${ }^{52}$ A. Sauret, D. Cébron, and M. Le Bars, "Spontaneous generation of inertial waves from boundary turbulence in a librating sphere,” J. Fluid Mech. 728, R5 (2013). 